\title{
A Well-Conditioned, Nonconforming Nitsche's Extended Finite Element Method for Elliptic Interface Problems
}

\author{
Xiaoxiao $\mathrm{He}^{1}$, Fei Song ${ }^{2}$ and Weibing Deng ${ }^{1, *}$ \\ ${ }^{1}$ Department of Mathematics, Nanjing University, Nanjing 210093, Jiangsu, \\ China \\ ${ }^{2}$ Department of Applied Mathematics, Nanjing Forestry University, Nanjing \\ 210037, Jiangsu, China
}

Received 25 March 2019; Accepted (in revised version) 6 July 2019

\begin{abstract}
In this paper, we introduce a nonconforming Nitsche's extended finite element method (NXFEM) for elliptic interface problems on unfitted triangulation elements. The solution on each side of the interface is separately expanded in the standard nonconforming piecewise linear polynomials with the edge averages as degrees of freedom. The jump conditions on the interface and the discontinuities on the cut edges (the segment of edges cut by the interface) are weakly enforced by the Nitsche's approach. In the method, the harmonic weighted fluxes are used and the extra stabilization terms on the interface edges and cut edges are added to guarantee the stability and the well conditioning. We prove that the convergence order of the errors in energy and $L^{2}$ norms are optimal. Moreover, the errors are independent of the position of the interface relative to the mesh and the ratio of the discontinuous coefficients. Furthermore, we prove that the condition number of the system matrix is independent of the interface position. Numerical examples are given to confirm the theoretical results.
\end{abstract}

AMS subject classifications: 65N12, 65N15, 65N30

Key words: Elliptic interface problems, NXFEM, nonconforming finite element, condition number.

\section{Introduction}

In this paper we consider the following elliptic interface problem:

$$
\begin{cases}-\nabla \cdot(a(x) \nabla u)=f & \text { in } \Omega_{1} \cup \Omega_{2}, \\ {[u]=g_{D}, \quad[(a(x) \nabla u) \cdot \mathbf{n}]=g_{N}} & \text { on } \Gamma, \\ u=0 & \text { on } \partial \Omega\end{cases}
$$

\footnotetext{
${ }^{*}$ Corresponding author. Email addresses: hxxmath@163.com (X. X. He), songfei@njfu.edu.cn (F. Song), wbdeng@nju.edu.cn (W. B. Deng) 


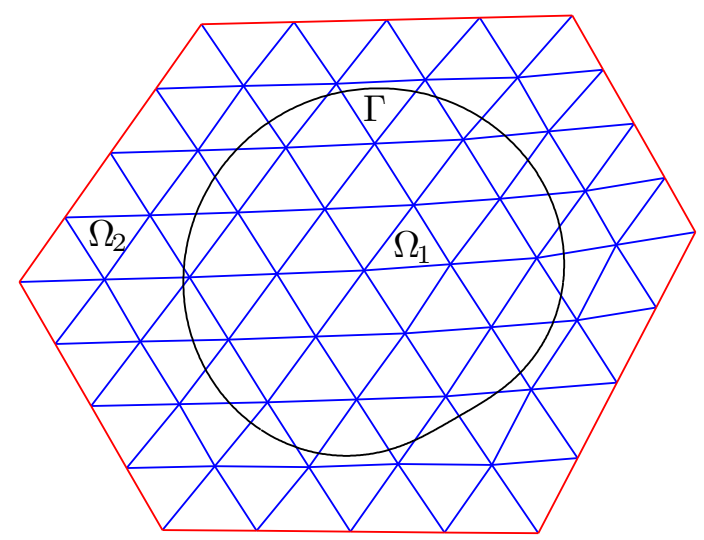

Figure 1: A sample domain $\Omega$ and an unfitted mesh.

where $\Omega=\Omega_{1} \cup \Gamma \cup \Omega_{2}$ is a bounded and convex domain in $\mathbb{R}^{2}, \Omega_{1}$ and $\Omega_{2}$ are two subdomains of $\Omega$ separated by the interface $\Gamma=\partial \Omega_{1} \cap \partial \Omega_{2}$ (see Fig. 1 for an illustration). $[v]=\left.v\right|_{\Omega_{1}}-\left.v\right|_{\Omega_{2}}$ denotes the jump of $v$ across the interface $\Gamma$, and $\mathbf{n}$ is the unit normal vector to $\Gamma$ pointing from $\Omega_{1}$ to $\Omega_{2}$. We assume that $a(x)=a_{i}$ for $x \in \Omega_{i}$ with constants $a_{i}>0, i=1,2$, and denote by $a_{\min }=\min _{i=1,2}\left\{a_{i}\right\}, a_{\max }=\max _{i=1,2}\left\{a_{i}\right\}$. We further assume that the interface $\Gamma$ is $C^{2}$-smooth.

The problem (1.1) is often occurred in material sciences and fluid dynamics which involves two or more distinct materials or fluids with different densities, conductivities or permeabilities. Much attention has been paid to the numerical methods for this problem in recent decades. We refer to the immersed boundary element method [1], the finite difference methods (see, for example, the immersed interface method [2] and the ghost fluid method [3]) and the finite element methods (see, for example, the multiscale finite element method [4], the immersed finite element method [5-10], the ghost fluid method [44], the splitting collocation method [43], the weak Galerkin finite element method $[42,45]$, the unfitted finite element method $[11-16,18]$ and the mortar element method [19]). In this paper, we focus on the numerical methods related to the finite element implementations.

Since the global regularity of the solution is low due to the nature of the interface and discontinuity of the coefficients in the equation, the performance of the standard finite element method is not very well unless the interface coincides with mesh lines. One strategy to solve the interface problems with accurate approximation is the interface-fitted grid methods where the discontinuity is directly captured by the mesh (see [19-24] and the references therein). However, it is difficult and time consuming to generate a body fitted grid for the interface problems with the complicated interface. In particular, for the moving interface problems, such a difficulty is more severe because of the expensive remeshing at each time step to maintain a good mesh. Therefore, various unfitted grid methods for the problem (1.1) have been proposed in the literature, where the interface can be arbitrarily located with respect to the mesh 
(see $[6-15,23,25,26]$ and the references therein).

In fact, the key point of the design of the numerical methods for the interface problems is the treatment of the discontinuities. Special techniques to incorporate the jump conditions on the interface with an unfitted grid method are needed. One way is the immersed finite element methods [2,5-10] where the finite element basis functions are locally modified for elements intersected by the interface to satisfy the jump conditions across the interface strongly or weakly. The other way is the Nitsche's extended finite element method (NXFEM) (also called the unfitted interface penalty finite element method or cut finite element method), in which the finite element space is enriched with extra basis functions across the interface and the discontinuity of the finite element space is then handled by the Nitsche's approach originally proposed for enforcing Dirichlet boundary condition through penalties [46]. This method was first proposed by Hansbo and Hansbo in [11] to solve the elliptic interface problem. Then a variety of related methods have been developed for different problems, such as elliptic interface problems $[12,13,15,16,18,25]$, Stokes interface problems $[27,28]$ and the Oseen problem [29].

For most of the NXFEMs, there are still two issues to consider. The first is whether the condition number of the system matrix is independent of the location of the interface relative to meshes. As shown in [30], the system matrix may be very illconditioned if the interface elements have very small cuts or the cut elements have very small intersection with the physical domain. To address this problem, Burman [30] proposed the classical Nitsche's method for the Possion's problem by adding a penalty term in the bilinear formulation that contained the difference between the solution and an $L^{2}$-projection of the solution on a patch of elements in the vicinity of the boundary. Further, Burman and Hansbo [31] proposed the fictitious domain Nitsche's method for the Possion equation with inhomogeneous Dirichlet conditions by adding a penalty term for normal-derivative jumps over the element face in the bilinear formulation. And then in [14], Wadbro et al. proposed a uniformly well-conditioned, unfitted Nitsche's method for the interface problems where the solutions on each side of the interface were enriched on the entire domain and the stabilized projection operators were involved in the bilinear formulation. Most of the above methods use the extra stabilization terms to guarantee the well conditioning. We remark that the preconditioning techniques has also been used in [32] to overcome the ill-conditioning. The second one is whether the error estimates are independent of the jump of the coefficients. To our knowledge, for most of the unfitted methods ([11-13, 15]) and even the above paper [14] to solve the interface problems, this issue is not clear. Consequently, some works have been introduced to ensure that the errors are independent of the ratio of discontinuous coefficients. For example, a modification of the NXFEM proposed in [11] was considered in [25], which is robust with respect to the coefficients. And a multiscale finite element method was proposed in [4] where it is proved that energy error estimates are independent of the contrast of the coefficients. Then, for high-contrast interface problems, Guzman, et al. [26] proposed a finite element method which defined local basis functions satisfying the continuity of the function and the continuity 
of the flux at certain points across the interface. They also added an extra stabilization term penalizing the jumps of the normal derivatives of the approximation across the edges cut by the interface. This idea was borrowed from the stabilized unfitted Nitsche method developed by Burman and Zunino in [17] and it is shown that the method introduced in [17] is robust with respect to diffusion contrast. Further, the Nitsche's method for the interface problems was introduced in [16] by adding the stabilized terms on some edges of elements cut by the interface. In a recent paper [18], the authors proposed an unfitted interface penalty finite element method which combined with the trick of the merging elements. This work has the following good features: the errors are optimal and independent of the location of the interface and the jump of the coefficient; in addition, the condition number of the system matrix is independent of the location of the interface with respect to the mesh.

However, there are few works using the nonconforming elements to solve the interface problems. It is well-known that the nonconforming elements on triangulation elements are used extensively due to their small stencil satisfying the inf-sup condition for Stokes equations. Therefore, it is necessary to analyze the nonconforming finite element method to solve the interface problems. We can refer to the works [9,33-35] for the nonconforming finite element method implementation. For example, the immersed finite element methods with the nonconforming elements on triangular meshes and rectangular meshes are proposed in [33] and [9] respectively. In [35], Capatina, et al. studied a nonconforming finite element method for an elliptic interface problem by adding some stabilization on the cut edges. It is shown that optimal a priori error estimates are robust with respect to the geometry and to the diffusion parameters. And then [34] presented the nonconforming Nitsche's extended finite element method on triangular meshes in two cases for an elliptic interface problem. One is the same as that method proposed in [35], and the other is obtained by modifying the nonconforming basis function on the cut elements. However, in these two papers the condition number of the system matrix has not been taken into consideration.

In this paper, we follow the partition in $[34,35]$, allowing elements cut by the interface and defining the enriched nonconforming finite element space. The difference from $[34,35]$ is that we use the weighted average flux only related to coefficients instead of that related to coefficients and subareas of the interface elements across the interface to guarantee the errors to be independent of the ratio of the discontinuous coefficients. This weighted average flux was used in [18] to solve elliptic interface problems with continuous piecewise finite elements. Comparing with the conforming elements, the nonconforming elements exhibit discontinuities across the local cut segments of the edges for the cut elements. Thus, special dispose for the cut segments is needed (see $[34,35]$ ). In this paper, we use the arithmetic average flux instead of the weighted average one on the cut segments used in $[34,35]$. Extra stabilization terms are added in the bilinear form, which adopt the techniques in $[16,26]$ (here we use different basis functions) to guarantee the stability and a well conditioning. In summary, we propose a nonconforming extended finite element method for the interface problems, which possesses all the good features of the work in [18]. Namely, the errors 
in the energy and $L^{2}$ norms are independent of the jump of the coefficients. Moreover, both the errors and the condition number of the stiffness matrix are independent of the interface position relative to the mesh.

The paper is organized as follows. In Section 2, we describe the Nitsche's extended finite element method with the nonconforming elements. In Section 3, we list some preliminary lemmas. The errors in the energy and $L^{2}$ norms are given in Section 4. In Section 5, we discuss the condition number of the discrete system. Numerical tests are presented in Section 6. Finally, we make a conclusion in Section 7.

Throughout the paper, $C, C_{1}, C_{2}, C_{i 1}, C_{i 2}, \cdots$ are used to denote the generic positive constants which are independent of $h$, the penalty parameters, and the jump of the coefficient $a(x)$. We also use the shorthand notation $A \lesssim B$ and $B \gtrsim A$ for the inequality $A \leq C B$ and $B \geq C A$.

\section{NXFEM with the nonconforming elements}

In this section, we give the formulation of the nonconforming Nitsche's extended finite element method.

First, we introduce some notations. Let $\left\{\mathcal{T}_{h}\right\}$ be a family of conforming, quasiuniform, and regular triangulations of the domain $\Omega$, which is independent of the position of $\Gamma$. Moreover, we need to make some assumptions concerning the intersection between $\Gamma$ and the mesh (see Assumptions (A1)-(A3) below). For any $K \in \mathcal{T}_{h}$, we define $h_{K}$ as $\operatorname{diam}(K)$ and denote $h:=\max _{K \in \mathcal{T}_{h}} h_{K}$. Note that any element $K \in \mathcal{T}_{h}$ is considered as closed. Define the set of cut elements by $G_{h}^{\Gamma}:=\left\{K \in \mathcal{T}_{h}: K \cap \Gamma \neq \emptyset\right\}$. In particular for $K \in G_{h}^{\Gamma}$, we denote $\Gamma_{K}=K \cap \Gamma$. Then we introduce $\mathcal{T}_{h}^{i}:=\left\{K \in \mathcal{T}_{h}\right.$ : $\left.K \cap \Omega_{i} \neq \emptyset\right\}$ and $\Omega_{h}^{i}:=\cup_{K \in \mathcal{T}_{h}^{i}} K$ for $i=1,2$. See Fig. 2 for an illustration of these definitions. Denote the set of all edges of $\mathcal{T}_{h}^{i}$ by $\varepsilon_{h}^{i}$. For each interior edge $e \in \varepsilon_{h}^{i}$, there are two elements $K_{l}$ and $K_{r}$ sharing the common side $e$, which is associated with a unit normal vector $\mathbf{n}_{e}$ pointing from $K_{l}$ to $K_{r}$.

In this paper, we make the following assumptions (see $[27,28]$ ):

(A1) It is assumed that the interface intersects the boundary of each triangle at most
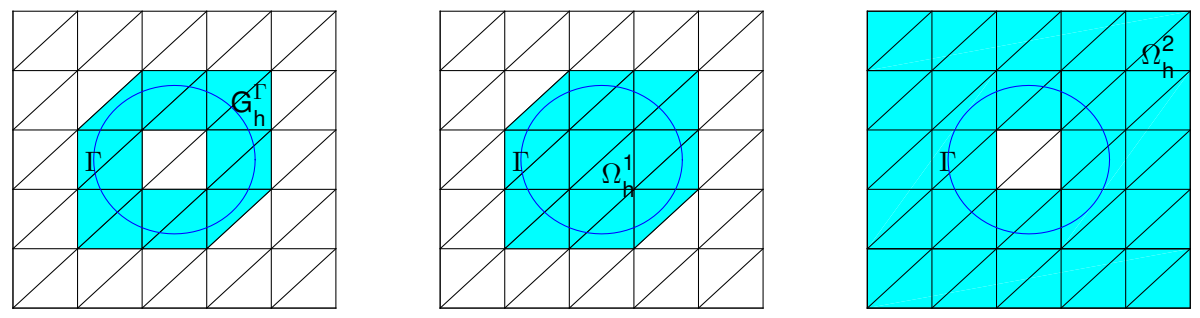

Figure 2: Illustration of definitions of set $G_{h}^{\Gamma}$ and sub-domains $\Omega_{h}^{1}$ and $\Omega_{h}^{2}$. Left figure: elements in $G_{h}^{\Gamma}$ (cobalt blue area). Center figure: sub-domain $\Omega_{h}^{1}$ (cobalt blue area). Right figure: sub-domain $\Omega_{h}^{2}$ (cobalt blue area). 
two points and each (open) edge at most once, or that the interface coincides with one edge of the element.

(A2) The interface is defined by the zeros isoline of a level set function. The level set function is then approximated by linear interpolation on the computational mesh. The interface is approximated by a chain of straight lines. That is to say, we assume that the straight line $\Gamma_{K, h}$ connecting the points of intersection between $\Gamma$ and $\partial K$ is a good approximation of $\Gamma_{K}$ for $K \in G_{h}^{\Gamma}$.

(A3) It is assumed that the mesh coincides with the outer boundary $\partial \Omega$.

Assumptions (A1) and (A2) state that the interface is well resolved by the mesh with an enough small mesh. Moreover, these two assumptions imply that the discrete approximation of the interface divides elements into simple shapes (two triangles or a triangle and a quadrilateral).

Before introducing the NXFEM space with nonconforming elements, we define the space

$$
V_{i}:=\left\{v \in L^{2}\left(\Omega_{h}^{i}\right): v \in H^{2}(K), \forall K \in \mathcal{T}_{h}^{i}\right\}, \quad i=1,2 .
$$

Further, we define a weak space:

$$
V:=\left\{v:\left.\left.v\right|_{\Omega_{i}} \in V_{i}\right|_{\Omega_{i}}, i=1,2,\left.v\right|_{\partial \Omega}=0\right\} .
$$

Denote the standard finite element space of nonconforming linear polynomials with support in $\Omega_{h}^{i}$ by

$$
\begin{aligned}
V_{h}^{i}:=\left\{v \in L^{2}\left(\Omega_{h}^{i}\right):\left.v\right|_{K} \in S_{h}(K) \text { if } K \in \mathcal{T}_{h}^{i} ;\right. \\
\quad \text { if } e=\partial K_{l} \cap \partial K_{r}, K_{l}, K_{r} \in \mathcal{T}_{h}^{i}, \text { then } \int_{e}[v] \mathrm{d} s=0 \\
\left.\quad \text { if } e=\partial K \cap \partial \Omega, K \in \mathcal{T}_{h}^{i}, \text { then } \int_{e} v \mathrm{~d} s=0\right\}, \quad i=1,2,
\end{aligned}
$$

where

$$
S_{h}(K):=\operatorname{span}\left\{\phi_{l}: \phi_{l} \in P_{1}(K), \frac{1}{\left|e_{m}\right|} \int_{e_{m}} \phi_{l} \mathrm{~d} s=\delta_{l m}, e_{m} \in \partial K, l, m=1,2,3\right\} .
$$

Then our nonconforming extended finite element space is defined by

$$
V_{h}:=\left\{v_{h}:\left.\left.v_{h}\right|_{\Omega_{i}} \in V_{h}^{i}\right|_{\Omega_{i}}, i=1,2\right\} .
$$

The set of uncut edges of $\mathcal{T}_{h}^{i}$ contained in $\Omega_{i}$ and the set of cut segments contained in $\Omega_{i}$ are also of interest. We denote them by $\varepsilon_{h}^{i, n c}$ and $\varepsilon_{h}^{i, c u t}$ respectively, which are defined as follows:

$$
\varepsilon_{h}^{i, n c}:=\left\{e \in \varepsilon_{h}^{i}: e=\partial K_{l} \cap \partial K_{r}, K_{l}, K_{r} \in \mathcal{T}_{h}^{i} \text { and } e \subset \Omega_{i}\right\},
$$



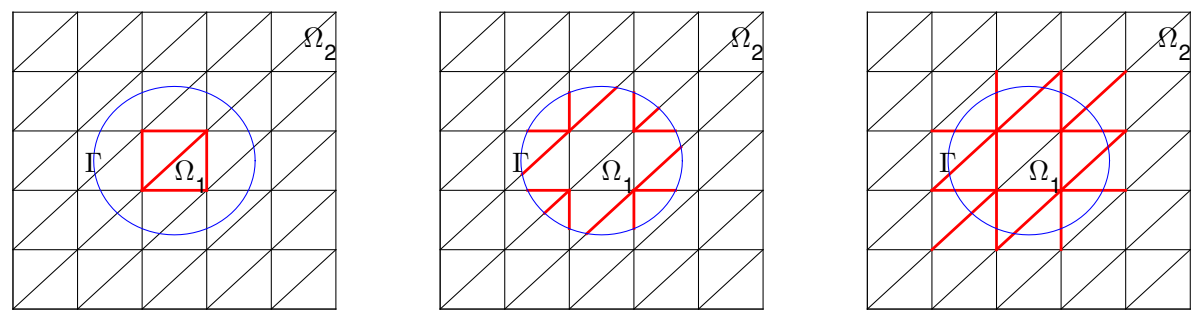

Figure 3: Illustration of definitions of set $\varepsilon_{h}^{1, n c}, \varepsilon_{h}^{1, c u t}$ and $\varepsilon_{h}^{\Gamma, 1}$. Left figure: edges in $\varepsilon_{h}^{1, n c}$ (red lines). Center figure: edges in $\varepsilon_{h}^{1, c u t}$ (red lines). Right figure: edges in $\varepsilon_{h}^{\Gamma, 1}$ (red lines).

and

$$
\varepsilon_{h}^{i, c u t}:=\left\{\widetilde{e}=e \cap \Omega_{i}: e=\partial K_{l} \cap \partial K_{r}, K_{l}, K_{r} \in G_{h}^{\Gamma}\right\} .
$$

That is to say, for each edge $\widetilde{e} \in \varepsilon_{h}^{i, c u t}$, there exist two cut elements $K_{l}, K_{r} \in G_{h}^{\Gamma}$ and $K_{j}^{i}=K_{j} \cap \Omega_{i}, j=l, r$ such that $\widetilde{e}=K_{l}^{i} \cap K_{r}^{i}$. Define jump and jump of the flux of $v \in V+V_{h}$ respectively by

$$
[v]=\left.v\right|_{K_{l}^{i}}-\left.v\right|_{K_{r}^{i}} \quad \text { and } \quad\left[\nabla v \cdot \mathbf{n}_{\widetilde{e}}\right]=\left.\nabla v\right|_{K_{l}^{i}} \cdot \mathbf{n}_{\widetilde{e}}-\left.\nabla v\right|_{K_{r}^{i}} \cdot \mathbf{n}_{\widetilde{e}},
$$

provided that $\mathbf{n}_{\widetilde{e}}$ is a unit normal vector to the edge $\widetilde{e}$ pointing from $K_{l}^{i}$ to $K_{r}^{i}$. Similarly, for $e \in \varepsilon_{h}^{i, n c}$, we can also define the jump of $v \in V+V_{h}$ on $e$ and a unit normal vector to the edge $e$ by $\mathbf{n}_{e}$. In particular, we note that $[v]=\left.v\right|_{K}$ for $e \in \varepsilon_{h}^{2, n c} \cap \partial \Omega$ and $e \subset \partial K$ with $K \in \mathcal{T}_{h}^{2}$. See Figs. 3(left and center) for an illustration of definitions of $\varepsilon^{1, n c}$ and $\varepsilon_{h}^{1, c u t}$ respectively.

Further, the set of all the edges of $G_{h}^{\Gamma}$ restricted to the interior of $\Omega_{h}^{i}$ is denoted by

$$
\varepsilon_{h}^{\Gamma, i}:=\left\{e=\partial K_{l} \cap \partial K_{r}: K_{l}, K_{r} \in \mathcal{T}_{h}^{i}, K_{l} \text { or } K_{r} \in G_{h}^{\Gamma}\right\} .
$$

Then we define jump $[\nabla v]=\left.\nabla v\right|_{K_{l}}-\left.\nabla v\right|_{K_{r}}$ for $v \in V+V_{h}$ on each edge $e \in \varepsilon_{h}^{\Gamma, i}$. See Fig. 3(right) for an illustration of the definition of $\varepsilon_{h}^{\Gamma, 1}$.

For any $v \in V+V_{h}$ and weights $w_{i} \in(0,1), i=1,2$, we define the averages $\{v\}_{w}$ and $\{v\}^{w}$ on the interface $\Gamma$ as follows:

$$
\{v\}_{w}=\left.w_{1} v_{1}\right|_{\Gamma}+\left.w_{2} v_{2}\right|_{\Gamma}, \quad\{v\}^{w}=\left.w_{2} v_{1}\right|_{\Gamma}+\left.w_{1} v_{2}\right|_{\Gamma}
$$

where $v_{i}=\left.v\right|_{\Omega_{i}}, i=1,2$. In this paper, we use the so-called "harmonic weights" as adopted by $[18,36]$,

$$
w_{1}=\frac{a_{2}}{a_{1}+a_{2}}, \quad w_{2}=\frac{a_{1}}{a_{1}+a_{2}} .
$$

Similarly, we denote the arithmetic weights $\{v\}_{k}$ on the cut edges $\widetilde{e} \in \varepsilon_{h}^{i, c u t}$ by

$$
\{v\}_{k}=\left.\frac{1}{2} v_{l}\right|_{\widetilde{e}}+\left.\frac{1}{2} v_{r}\right|_{\widetilde{e}}
$$


where $v_{j}=\left.v\right|_{K_{j}}, j=l, r, K_{l}, K_{r} \in G_{h}^{\Gamma}$. We now give the nonconforming NXFEM based on the weak formulation of problem (1.1), penalty terms of the jump across the interface and the jump across the cut segments contained in $\Omega_{i}$, and stabilization terms on edges in $\varepsilon_{h}^{\Gamma, i}$ and $\varepsilon_{h}^{i, c u t}, i=1,2$. The nonconforming NXFEM is to find $u_{h} \in V_{h}$ such that

$$
A_{h}\left(u_{h}, v_{h}\right)=L\left(v_{h}\right), \quad \forall v_{h} \in V_{h},
$$

where $A_{h}(\cdot, \cdot)$ is the bilinear form on $\left(V+V_{h}\right) \times\left(V+V_{h}\right)$ and $L(\cdot)$ is the linear functional on $V+V_{h}$ defined by

$$
\begin{aligned}
A_{h}(u, v)=\sum_{i=1}^{2} & \sum_{K \in \mathcal{T}_{h}^{i}} \int_{K \cap \Omega_{i}} a_{i} \nabla u \cdot \nabla v-\sum_{K \in G_{h}^{\Gamma}} \int_{\Gamma_{K}}\left(\{a \nabla u \cdot \mathbf{n}\}_{w}[v]\right. \\
& \left.+[u]\{a \nabla v \cdot \mathbf{n}\}_{w}\right)+\frac{\gamma_{0}\{a\}_{w}}{h} \sum_{K \in G_{h}^{\Gamma}} \int_{\Gamma_{K}}[u][v] \\
& -\sum_{i=1}^{2} \sum_{\widetilde{e} \in \varepsilon_{h}^{i, c u t}}\left(\int_{\widetilde{e}}\left(\left\{a_{i} \nabla u \cdot \mathbf{n}_{\widetilde{e}}\right\}_{k}[v]+\left\{a_{i} \nabla v \cdot \mathbf{n}_{\widetilde{e}}\right\}_{k}[u]\right)\right. \\
& \left.-\frac{\gamma_{i} a_{i}}{|\widetilde{e}|} \int_{\widetilde{e}}[u][v]\right)+J^{0}(u, v)+J^{1}(u, v),
\end{aligned}
$$

with

$$
\begin{aligned}
& J^{0}(u, v)=\sum_{i=1}^{2} \sum_{e \in \varepsilon_{h}^{\Gamma, i}}|e| a_{i} \int_{e}[\nabla u] \cdot[\nabla v], \\
& J^{1}(u, v)=\sum_{i=1}^{2} \sum_{\widetilde{e} \in \varepsilon_{h}^{i, c u t}}|\widetilde{e}| a_{i} \int_{\widetilde{e}}\left[\nabla u \cdot \mathbf{n}_{\widetilde{e}}\right]\left[\nabla v \cdot \mathbf{n}_{\widetilde{e}}\right],
\end{aligned}
$$

and

$$
\begin{aligned}
L(v)=\sum_{i=1}^{2} & \int_{\Omega_{i}} f v+\sum_{K \in G_{h}^{\Gamma}} \int_{\Gamma_{K}} g_{N}\{v\}^{w} \\
& -\sum_{K \in G_{h}^{\Gamma}} \int_{\Gamma_{K}} g_{D}\{a \nabla v \cdot \mathbf{n}\}_{w}+\frac{\gamma_{0}\{a\}_{w}}{h} \sum_{K \in G_{h}^{\Gamma}} \int_{\Gamma_{K}} g_{D}[v],
\end{aligned}
$$

and $\gamma_{0}, \gamma_{1}$ and $\gamma_{2}$ are positive parameters to be chosen later.

Let $H^{2}\left(\Omega_{1} \cup \Omega_{2}\right)$ be the piecewise $H^{2}$ space such that if $v \in H^{2}\left(\Omega_{1} \cup \Omega_{2}\right)$ then $\left.v\right|_{\Omega_{1}} \in$ $H^{2}\left(\Omega_{1}\right)$ and $\left.v\right|_{\Omega_{2}} \in H^{2}\left(\Omega_{2}\right)$. For the solution to the interface problem (1.1) which satisfies $u \in H^{2}\left(\Omega_{1} \cup \Omega_{2}\right)$, it is easy to check that it satisfies the following equation:

$$
A_{h}(u, v)=\sum_{i=1}^{2} \sum_{e \in \varepsilon_{h}^{i, n c}} \int_{e} a_{i} \nabla u \cdot \mathbf{n}_{e}[v]+L(v), \quad \forall v \in V_{h} .
$$


To end this section, we introduce the following energy norm on the space $V+V_{h}$ :

$$
\begin{aligned}
\|v\| \|^{2}:=\sum_{i=1}^{2} & \sum_{K \in \mathcal{T}_{h}^{i}}\left\|\sqrt{a_{i}} \nabla v\right\|_{L^{2}\left(K \cap \Omega_{i}\right)}^{2}+\frac{\{a\}_{w}}{h} \sum_{K \in G_{h}^{\Gamma}}\|[v]\|_{L^{2}\left(\Gamma_{K}\right)}^{2} \\
& +\sum_{i=1}^{2} \sum_{e \in \varepsilon_{h}^{\Gamma, i}}|e| a_{i}\|[\nabla v]\|_{L^{2}(e)}^{2} \\
& +\sum_{i=1}^{2} \sum_{\widetilde{e} \in \varepsilon_{h}^{i, c u t}}\left(|\widetilde{e}| a_{i}\left\|\left[\nabla v \cdot \mathbf{n}_{\widetilde{e}}\right]\right\|_{L^{2}(\widetilde{e})}^{2}+|\widetilde{e}|^{-1} a_{i}\|[v]\|_{L^{2}(\widetilde{e})}^{2}\right) .
\end{aligned}
$$

\section{Some preliminary lemmas}

In this section, we give some useful lemmas. Recalling the partition of the domain and Assumptions (A1) and (A2) in Section 2, we have the following Lemmas 3.13.3, where Lemma 3.1, Lemma 3.2 and Lemma 3.3 are proved in [16, 26] and [13], respectively.

Lemma 3.1. Consider a node $z$ of the triangulation $\mathcal{T}_{h}^{i}$ such that $z \in \Omega_{i}$. Let $\Delta_{z}$ be the patch of elements associated to $z$, i.e., $\Delta_{z}=\cup\left\{K: K \in \mathcal{T}_{h}^{i}, z \in \partial K\right\}$. Under Assumptions (A1) and (A2), there exists an element $K_{z} \in \Delta_{z}$ such that

$$
\left|K_{z} \cap \Omega_{i}\right| \geq C h_{K_{z}}^{2}
$$

Lemma 3.2. If $\widetilde{e} \in \varepsilon_{h}^{i, c u t}$, that is to say, $\widetilde{e}=\partial K_{l}^{i} \cap \partial K_{r}^{i}$, where $K_{l}, K_{r} \in G_{h}^{\Gamma}$ and $K_{j}^{i}=K_{j} \cap \Omega_{i}, j=l, r$, and Assumptions (A1) and (A2) hold, then there exists a constant $\theta>0$ such that

$$
|\widetilde{e}|^{2} \leq \theta \max _{j=l, r}\left|K_{j}^{i}\right|
$$

The constant $\theta$ depends on the shape regularity of $K_{l}$ and $K_{r}$, and the $C^{2}$-norm of the parametrization of the interface.

Lemma 3.3. If Assumptions (A1) and (A2) hold, then for each $K \in G_{h}^{\Gamma}$ and $v \in H^{1}(K)$, it holds

$$
\|v\|_{L^{2}\left(\Gamma_{K}\right)} \lesssim h_{K}^{-1 / 2}\|v\|_{L^{2}(K)}+\|v\|_{L^{2}(K)}^{1 / 2}\|\nabla v\|_{L^{2}(K)}^{1 / 2} .
$$

Further, if $v \in P_{1}(K)$, then

$$
\|v\|_{L^{2}\left(\Gamma_{K}\right)} \lesssim h_{K}^{-1 / 2}\|v\|_{L^{2}(K)} .
$$

In order to estimate the error of our method, we need the following trace inequality for the cut segments contained in $\Omega_{i}$. 
Lemma 3.4. Suppose that $v \in H^{2}(K)$ for $K \in G_{h}^{\Gamma}$. If $e \subset \partial K$ and $\widetilde{e} \in \varepsilon_{h}^{i, c u t}$ such that $\widetilde{e} \subseteq$, then we have

$$
\frac{1}{|\widetilde{e}|}\|v\|_{L^{2}(\widetilde{e})}^{2} \leq C\left(\frac{1}{h_{K}^{2}}\|v\|_{L^{2}(K)}^{2}+\|\nabla v\|_{L^{2}(K)}^{2}+h_{K}^{2}|\nabla v|_{H^{1}(K)}^{2}\right) .
$$

Proof. Since $\widetilde{e} \subseteq e$, from Lemma 3 of [37], we have

$$
\frac{1}{|\widetilde{e}|}\|v\|_{L^{2}(\widetilde{e})}^{2} \leq C\left(\frac{1}{|e|}\|v\|_{L^{2}(e)}^{2}+|e|\|\nabla v\|_{L^{2}(e)}^{2}\right)
$$

then using the trace inequality yields the result.

The following well known extension result plays an important role in the following error analysis (see [38]).

Lemma 3.5. There exist two extension operators $E_{1}: H^{2}\left(\Omega_{1}\right) \rightarrow H^{2}(\Omega) \cap H_{0}^{1}(\Omega)$ and $E_{2}: H^{2}\left(\Omega_{2}\right) \cap H_{0}^{1}(\Omega) \rightarrow H^{2}(\Omega) \cap H_{0}^{1}(\Omega)$ such that

$$
\left.\left(E_{i} v\right)\right|_{\Omega_{i}}=v, \quad\left\|E_{i} v\right\|_{H^{j}(\Omega)} \lesssim\|v\|_{H^{j}\left(\Omega_{i}\right)}, \quad i=1,2, \quad j=0,1,2,
$$

where $v \in H^{j}\left(\Omega_{i}\right)$ for $i=1$ and $v \in H^{j}\left(\Omega_{i}\right),\left.v\right|_{\partial \Omega}=0$ for $i=2$.

\section{Error estimates for the nonconforming NXFEM}

In this section, we derive the error estimates of the energy and $L^{2}$ norms. First, we give the coercivity of the bilinear form $A_{h}(\cdot, \cdot)$.

Lemma 4.1 (Coercivity). Assume that $\gamma_{0}, \gamma_{1}$ and $\gamma_{2}$ are large enough. Under Assumptions (A1) and (A2), the following inequality holds

$$
A_{h}(v, v) \geq \frac{1}{2}\|v\|^{2}, \quad \forall v \in V_{h} .
$$

Proof. Denote $v_{i}=\left.v\right|_{\Omega_{i}}, i=1,2$. From the definition of $A_{h}(\cdot, \cdot)$, we have

$$
\begin{aligned}
A_{h}(v, v)=\sum_{i=1}^{2} & \sum_{K \in \mathcal{T}_{h}^{i}}\left\|\sqrt{a_{i}} \nabla v\right\|_{L^{2}\left(K \cap \Omega_{i}\right)}^{2}-2 \sum_{K \in G_{h}^{\Gamma}} \int_{\Gamma_{K}}\{a \nabla v \cdot \mathbf{n}\}_{w}[v] \\
& +\frac{\gamma_{0}\{a\}_{w}}{h} \sum_{K \in G_{h}^{\Gamma}}\|[v]\|_{L^{2}\left(\Gamma_{K}\right)}^{2} \\
& +\sum_{i=1}^{2} \sum_{e \in \varepsilon_{h}^{\Gamma, i}} a_{i}|e|\|[\nabla v]\|_{L^{2}(e)}^{2}-2 \sum_{i=1}^{2} \sum_{\widetilde{e} \in \varepsilon_{h}^{i, c u t}} \int_{\widetilde{e}}\left\{a_{i} \nabla v \cdot \mathbf{n}_{\widetilde{e}}\right\}_{k}[v] \\
& +\sum_{i=1}^{2} \sum_{\widetilde{e} \in \varepsilon_{h}^{i, c u t}}\left(\gamma_{i} a_{i}|\widetilde{e}|^{-1}\|[v]\|_{L^{2}(\widetilde{e})}^{2}+a_{i}|\widetilde{e}|\left\|\left[\nabla v \cdot \mathbf{n}_{\widetilde{e}}\right]\right\|_{L^{2}(\widetilde{e})}^{2}\right) .
\end{aligned}
$$




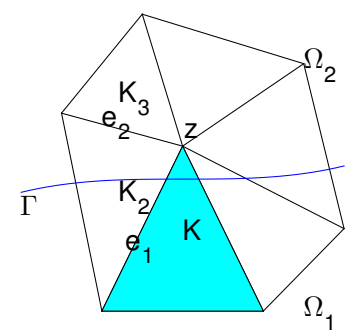

Figure 4: Illustration of the definition of $\Delta_{z}$.

To prove the coercivity, it is sufficient to bound the two non-symmetric terms. For the first non-symmetric term, using the Cauchy-Schwarz and Young inequalities, we obtain

$$
2\left|\int_{\Gamma_{K}}\{a \nabla v \cdot \mathbf{n}\}_{w}[v]\right| \leq \frac{h}{\{a\}_{w} \epsilon}\left\|\{a \nabla v \cdot \mathbf{n}\}_{w}\right\|_{L^{2}\left(\Gamma_{K}\right)}^{2}+\frac{\{a\}_{w} \epsilon}{h}\|[v]\|_{L^{2}\left(\Gamma_{K}\right)}^{2},
$$

where $\epsilon$ is a positive number to be fixed later.

Now we estimate the first term of the right-hand side of (4.1). It is easy to see that

$$
\begin{aligned}
& \frac{h}{\{a\}_{w}}\left\|\{a \nabla v \cdot \mathbf{n}\}_{w}\right\|_{L^{2}\left(\Gamma_{K}\right)}^{2} \leq \sum_{i=1}^{2} 2 h \frac{a_{i}^{2} w_{i}^{2}}{\{a\}_{w}}\left\|\nabla v_{i} \cdot \mathbf{n}\right\|_{L^{2}\left(\Gamma_{K}\right)}^{2} \\
\leq & h\left(\left\|\sqrt{a_{1}} \nabla v_{1} \cdot \mathbf{n}\right\|_{L^{2}\left(\Gamma_{K}\right)}^{2}+\left\|\sqrt{a_{2}} \nabla v_{2} \cdot \mathbf{n}\right\|_{L^{2}\left(\Gamma_{K}\right)}^{2}\right),
\end{aligned}
$$

where we have used the fact that

$$
\frac{a_{i}^{2} w_{i}^{2}}{\{a\}_{w}}<\frac{1}{2} a_{i}, \quad i=1,2 .
$$

Let $K \in G_{h}^{\Gamma}$, and let $z \in \Omega_{i}$ be a node of $K$. According to Lemma 3.1, if $h$ is small enough, then there exist an element $K_{z} \in \Delta_{z}$ and a constant $C_{z}$ such that $\left|K_{z} \cap \Omega_{i}\right| \geq$ $C_{z} h_{K_{z}}^{2}$. We assume that

$$
e_{1} \subset \partial K, \quad e_{1}, e_{2} \subset \partial K_{2}, \cdots, e_{n-1}, e_{n} \subset \partial K_{z},
$$

where $K, K_{2}, \cdots, K_{z} \in \Delta_{z}$. Namely, $e_{1}=\partial K \cap \partial K_{2}, e_{2}=\partial K_{2} \cap \partial K_{3}, \cdots, e_{n-1}=$ $\partial K_{n-1} \cap \partial K_{z}$ (see Fig. 4). Then, using the fact that $\nabla v_{i}$ is a constant vector in each element, we have

$$
\begin{aligned}
h\left\|\sqrt{a_{i}} \nabla v_{i} \cdot \mathbf{n}\right\|_{L^{2}\left(\Gamma_{K}\right)}^{2} & \leq \frac{h\left|\Gamma_{K}\right|}{\left|e_{1}\right|}\left\|\left.\sqrt{a_{i}} \nabla v_{i}\right|_{K}\right\|_{L^{2}\left(e_{1}\right)}^{2} \leq C_{i 1}\left|e_{1}\right|\left\|\left.\sqrt{a_{i}} \nabla v_{i}\right|_{K}\right\|_{L^{2}\left(e_{1}\right)}^{2} \\
& \leq C_{i 1}\left|e_{1}\right|\left(a_{i}\left\|\left[\nabla v_{i}\right]\right\|_{L^{2}\left(e_{1}\right)}^{2}+\left\|\sqrt{a_{i}} \nabla v_{i} \mid K_{K_{2}}\right\|_{L^{2}\left(e_{1}\right)}^{2}\right) \\
& \leq C_{i 1}\left|e_{1}\right| a_{i}\left\|\left[\nabla v_{i}\right]\right\|_{L^{2}\left(e_{1}\right)}^{2}+C_{i 1} C_{i 2}\left|e_{2}\right|\left\|\left.\sqrt{a_{i}} \nabla v_{i}\right|_{K_{2}}\right\|_{L^{2}\left(e_{2}\right)}^{2}
\end{aligned}
$$


where we have used the fact that $\frac{h\left|\Gamma_{K}\right|}{\left|e_{1}\right|^{2}} \leq C_{i 1}$ because of the mesh is quasi-uniform and $\frac{\left|e_{1}\right|^{2}}{\left|e_{2}\right|^{2}} \leq C_{i 2}$ since the triangulation is regular. Further, using the following fact that

$$
\left\|\left.\sqrt{a_{i}} \nabla v_{i}\right|_{K_{2}}\right\|_{L^{2}\left(e_{2}\right)}^{2} \leq a_{i}\left\|\left[\nabla v_{i}\right]\right\|_{L^{2}\left(e_{2}\right)}^{2}+\left\|\left.\sqrt{a_{i}} \nabla v_{i}\right|_{K_{3}}\right\|_{L^{2}\left(e_{2}\right)}^{2},
$$

we have

$$
\begin{gathered}
h\left\|\sqrt{a_{i}} \nabla v_{i} \cdot \mathbf{n}\right\|_{L^{2}\left(\Gamma_{K}\right)}^{2} \leq C_{i 1}\left|e_{1}\right| a_{i}\left\|\left[\nabla v_{i}\right]\right\|_{L^{2}\left(e_{1}\right)}^{2}+C_{i 1} C_{i 2}\left|e_{2}\right| a_{i}\left\|\left[\nabla v_{i}\right]\right\|_{L^{2}\left(e_{2}\right)}^{2} \\
+C_{i 1} C_{i 2} C_{i 3}\left|e_{3}\right|\left\|\left.\sqrt{a_{i}} \nabla v_{i}\right|_{K_{3}}\right\|_{L^{2}\left(e_{3}\right)}^{2},
\end{gathered}
$$

where we have used the fact that $\frac{\left|e_{2}\right|^{2}}{\left|e_{3}\right|^{2}} \leq C_{i 3}$. Thus, by the same argument, we obtain

$$
\begin{aligned}
& h\left\|\sqrt{a_{i}} \nabla v_{i} \cdot \mathbf{n}\right\|_{L^{2}\left(\Gamma_{K}\right)}^{2} \\
\leq & \sum_{j=1}^{n-1} C_{i 1} \cdots C_{i j}\left|e_{j}\right| a_{i}\left\|\left[\nabla v_{i}\right]\right\|_{L^{2}\left(e_{j}\right)}^{2}+C_{i 1} \cdots C_{i n}\left|e_{n}\right|\left\|\left.\sqrt{a_{i}} \nabla v_{i}\right|_{K_{z}}\right\|_{L^{2}\left(e_{n}\right)}^{2} \\
\leq & \sum_{j=1}^{n-1} C_{i 1} \cdots C_{i j}\left|e_{j}\right| a_{i}\left\|\left[\nabla v_{i}\right]\right\|_{L^{2}\left(e_{j}\right)}^{2}+C_{i 1} \cdots C_{i n} \frac{\left|e_{n}\right|^{2}}{C_{z} h_{K_{z}}^{2}}\left\|\left.\sqrt{a_{i}} \nabla v_{i}\right|_{K_{z}}\right\|_{L^{2}\left(K_{z} \cap \Omega_{i}\right)}^{2} \\
\leq & C_{i}\left(\left\|\sqrt{a_{i}} \nabla v\right\|_{L^{2}\left(K_{z} \cap \Omega_{i}\right)}^{2}+\sum_{e \in \varepsilon_{h}^{\Gamma, i}} a_{i}|e|\|[\nabla v]\|_{L^{2}(e)}^{2}\right)
\end{aligned}
$$

Next, we bound

$$
2 \sum_{i=1}^{2} \sum_{\widetilde{e} \in \varepsilon_{h}^{i, c u t}} \int_{\widetilde{e}}\left\{a_{i} \nabla v \cdot \mathbf{n}_{\widetilde{e}}\right\}_{k}[v] .
$$

Note that any $\widetilde{e} \in \varepsilon_{h}^{i, c u t}$ is the cut segment. Let $K_{l}, K_{r} \in G_{h}^{\Gamma}$ and $K_{l}^{i}=K_{l} \cap \Omega_{i}$, $K_{r}^{i}=K_{r} \cap \Omega_{i}$ satisfy $\widetilde{e}=\partial K_{l}^{i} \cap \partial K_{r}^{i}$. Without loss of generality, we assume $\left|K_{l}^{i}\right|=$ $\max _{j=l, r}\left|K_{j}^{i}\right|$. According to Lemma 3.2, it holds

$$
|\widetilde{e}|^{2} \leq \theta\left|K_{l}^{i}\right|
$$

It is easy to see

$$
\sum_{\widetilde{e} \in \varepsilon_{h}^{i, c u t}} \int_{\widetilde{e}}\left\{a_{i} \nabla v \cdot \mathbf{n}_{\widetilde{e}}\right\}_{k}[v]=\sum_{\widetilde{e} \in \varepsilon_{h}^{i, c u t}} \int_{\widetilde{e}} a_{i}\left(\left.\nabla v\right|_{K_{l}} \cdot \mathbf{n}_{\widetilde{e}} \pm \frac{1}{2}\left[\nabla v \cdot \mathbf{n}_{\widetilde{e}}\right]\right)[v] .
$$

Using the fact that $\nabla v$ is a constant vector, from (4.6), we have

$$
\begin{aligned}
& \left.\int_{\widetilde{e}} a_{i} \nabla v\right|_{K_{l}} \cdot \mathbf{n}_{\widetilde{e}}[v] \\
\leq & \sqrt{\theta}\left\|\sqrt{a_{i}} \nabla v\right\|_{L^{2}\left(K_{l}^{i}\right)} \frac{\sqrt{a_{i}}}{\sqrt{|\widetilde{e}|}}\|[v]\|_{L^{2}(\widetilde{e})} \\
\leq & \frac{\theta}{2 \epsilon}\left\|\sqrt{a_{i}} \nabla v\right\|_{L^{2}\left(K_{l}^{i}\right)}^{2}+\frac{\epsilon}{2|\widetilde{e}|} a_{i}\|[v]\|_{L^{2}(\widetilde{e})}^{2} .
\end{aligned}
$$


A Well-Conditioned, Nonconforming Nitsche's Extended Finite Element Method

Further, using the Cauchy-Schwarz inequality, we have

$$
\int_{\widetilde{e}} a_{i}\left[\nabla v \cdot \mathbf{n}_{\widetilde{e}}\right][v] \leq \frac{1}{2} a_{i}|\widetilde{e}|\left\|\left[\nabla v \cdot \mathbf{n}_{\widetilde{e}}\right]\right\|_{L^{2}(\widetilde{e})}^{2}+\frac{1}{2} a_{i}|\widetilde{e}|^{-1}\|[v]\|_{L^{2}(\widetilde{e})}^{2} \cdot
$$

Then, collecting the above two estimates (4.8) and (4.9), we obtain

$$
\begin{aligned}
& 2 \int_{\widetilde{e}} a_{i}\left(\left.\nabla v\right|_{K_{l}} \cdot \mathbf{n}_{\widetilde{e}} \pm \frac{1}{2}\left[\nabla v \cdot \mathbf{n}_{\widetilde{e}}\right]\right)[v] \\
\leq & \frac{\theta}{\epsilon}\left\|\sqrt{a_{i}} \nabla v\right\|_{L^{2}\left(K_{l}^{i}\right)}^{2}+\frac{1}{2} a_{i}|\widetilde{e}|\left\|\left[\nabla v \cdot \mathbf{n}_{\widetilde{e}}\right]\right\|_{L^{2}(\widetilde{e})}^{2}+\frac{\epsilon+1 / 2}{|\widetilde{e}|} a_{i}\|[v]\|_{L^{2}(\widetilde{e})}^{2} .
\end{aligned}
$$

Therefore,

$$
\begin{aligned}
A_{h}(v, v) \geq \sum_{i=1}^{2} & \sum_{K \in \mathcal{T}_{h}^{i}}\left(1-\frac{C_{i}+\theta}{\epsilon}\right)\left\|\sqrt{a_{i}} \nabla v\right\|_{L^{2}\left(K \cap \Omega_{i}\right)}^{2} \\
& +\frac{\left(\gamma_{0}-\epsilon\right)\{a\}_{w}}{h} \sum_{K \in G_{h}^{\Gamma}}\|[v]\|_{L^{2}\left(\Gamma_{K}\right)}^{2}+\sum_{i=1}^{2} \sum_{e \in \varepsilon_{h}^{\Gamma, i}}\left(1-\frac{C_{i}}{\epsilon}\right) a_{i}|e|\|[\nabla v]\|_{L^{2}(e)}^{2} \\
& +\sum_{i=1}^{2} \sum_{\widetilde{e} \in \varepsilon_{h}^{i, c u t}}\left(\frac{\gamma_{i}-\epsilon-1 / 2}{|\widetilde{e}|} a_{i}\|[v]\|_{L^{2}(\widetilde{e})}^{2}+\frac{a_{i}|\widetilde{e}|}{2}\left\|\left[\nabla v \cdot \mathbf{n}_{\widetilde{e}}\right]\right\|_{L^{2}(\widetilde{e})}^{2}\right) .
\end{aligned}
$$

Taking $\epsilon=2\left(\max \left\{C_{1}, C_{2}\right\}+\theta\right)$, we can conclude the result by choosing

$$
\gamma_{0}>\varepsilon+1 / 2, \quad \gamma_{i}>\varepsilon+1, \quad i=1,2 .
$$

This completes the proof.

Next, we prove the continuity of the bilinear form. To do this, we define the augmented norm as follows:

$$
\begin{aligned}
\|v\|_{V}^{2}:=\|v\|^{2} & +\frac{h}{\{a\}_{w}} \sum_{K \in G_{h}^{\Gamma}}\left\|\{a \nabla v \cdot n\}_{w}\right\|_{L^{2}\left(\Gamma_{K}\right)}^{2} \\
& +\sum_{i=1}^{2} \sum_{\widetilde{e} \in \varepsilon_{h}^{i, c u t}} a_{i}|\widetilde{e}|\left\|\left\{\nabla v \cdot \mathbf{n}_{\widetilde{e}}\right\}_{k}\right\|_{L^{2}(\widetilde{e})}^{2} .
\end{aligned}
$$

Lemma 4.2 (Continuity). For any $u, v \in V$, there exists a constant $C>0$ independent of $u, v$ such that

$$
A_{h}(u, v) \leq C\||u|\|_{V}\||v|\|_{V} .
$$

Additionally, for $u \in V$ and $v \in V_{h}$, under Assumptions (A1) and (A2), it holds

$$
A_{h}(u, v) \leq C\|\| u\left\|_{V}\right\|\|v\| \| .
$$

Moreover, for $v \in V_{h}$, under Assumptions (A1) and (A2), the following inequality holds

$$
\|v\|_{V} \leq C\||v|\| \text {. }
$$


Proof. (4.11) can be obtained by the Cauchy-Schwarz inequality easily. Then we only need to prove (4.13) which can infer (4.12) directly.

Now we prove (4.13). From (4.2) and (4.5), we have

$$
\begin{aligned}
& \frac{h}{\{a\}_{w}} \sum_{K \in G_{h}^{\Gamma}}\left\|\{a \nabla v \cdot \mathbf{n}\}_{w}\right\|_{L^{2}\left(\Gamma_{K}\right)}^{2} \\
\leq & \sum_{i=1}^{2} \sum_{K \in G_{h}^{\Gamma}} a_{i} h\|\nabla v \cdot \mathbf{n}\|_{L^{2}\left(\Gamma_{K}\right)}^{2} \lesssim\|v\| \|^{2} .
\end{aligned}
$$

We also need to bound

$$
\sum_{i=1}^{2} \sum_{\widetilde{e} \in \varepsilon_{h}^{i, c u t}} a_{i}|\widetilde{e}|\left\|\left\{\nabla v \cdot \mathbf{n}_{\widetilde{e}}\right\}_{k}\right\|_{L^{2}(\widetilde{e})}^{2} .
$$

Similar to the analysis of Lemma 4.1 for any $\widetilde{e} \in \varepsilon_{h}^{i, c u t}$, we let $K_{l}, K_{r} \in G_{h}^{\Gamma}$ and $K_{l}^{i}=$ $K_{l} \cap \Omega_{i}, K_{r}^{i}=K_{r} \cap \Omega_{i}$ satisfy $\widetilde{e}=\partial K_{l}^{i} \cap \partial K_{r}^{i}$, and assume $\left|K_{l}^{i}\right|=\max _{j=l, r}\left|K_{j}^{i}\right|$. According to Lemma 3.2, $|\widetilde{e}|^{2} \leq \theta\left|K_{l}^{i}\right|$ holds. Then

$$
\begin{aligned}
& a_{i}|\widetilde{e}|\left\|\left\{\nabla v \cdot \mathbf{n}_{\widetilde{e}}\right\}_{k}\right\|_{L^{2}(\widetilde{e})}^{2} \\
\leq & a_{i}|\widetilde{e}| \sum_{j=l, r}\left\|\left.\nabla v\right|_{K_{j}^{i}}\right\|_{L^{2}(\widetilde{e})}^{2} \\
\lesssim & a_{i}|\widetilde{e}|\left(\left.|| \nabla v\right|_{K_{l}^{i}}\left\|_{L^{2}(\widetilde{e})}^{2}+\right\|[\nabla v] \|_{L^{2}(\widetilde{e})}^{2}\right) \\
\lesssim & \left\|\sqrt{a_{i}} \nabla v\right\|_{L^{2}\left(K_{l}^{i}\right)}^{2}+a_{i}|\widetilde{e}|\|[\nabla v]\|_{L^{2}(\widetilde{e})}^{2}
\end{aligned}
$$

Thus, by use of the definitions of $\|v\|_{V}$ and $\|v\| \|$, we obtain (4.13).

To give the energy error estimate, we construct an interpolation operator $I_{h}$ with the integral-value as degrees of freedom. For any $v \in H^{2}\left(\Omega_{1} \cup \Omega_{2}\right)$, denote by $v_{i}=\left.v\right|_{\Omega_{i}}$, $i=1,2$, and take the interpolant $I_{h} v$ of $v$ onto $V_{h}$, which is defined as

$$
\left.\left(I_{h} v\right)\right|_{\Omega_{i}}=\left.\left(I_{h} v_{i}\right)\right|_{\Omega_{i}}, \quad I_{h} v_{i}=\Pi_{h}\left(\left.E_{i} v_{i}\right|_{\Omega_{h}^{i}}\right) \in V_{h}^{i}, \quad i=1,2,
$$

where $E_{i}$ is defined in Lemma 3.5 and $\Pi_{h}$ is the standard Crouzeix-Raviart interpolation operator. The optimal approximation error estimate is shown below.

Lemma 4.3. Suppose that $v \in H^{2}\left(\Omega_{1} \cup \Omega_{2}\right)$ and $I_{h} v$ is the interpolation defined by (4.14). Then

$$
\left\|\left|v-I_{h} v\right|\right\|_{V} \lesssim h\left(\sqrt{a_{1}}|v|_{H^{2}\left(\Omega_{1}\right)}+\sqrt{a_{2}}|v|_{H^{2}\left(\Omega_{2}\right)}\right) .
$$

Proof. Denote by $\eta_{i}=\left.E_{i} v_{i}\right|_{\Omega_{h}^{i}}-I_{h} v_{i}, i=1,2$ and by $\eta=v-I_{h} v$. From the standard finite element interpolation theory in [39] and Lemma 3.5, for $i=1,2, j=0,1,2$, we have

$$
\left\|\eta_{i}\right\|_{H^{j}(K)} \lesssim h^{s-j}\left|E_{i} v_{i}\right|_{H^{s}(K)}, \quad j \leq s \leq 2
$$


and

$$
\sum_{K \in \mathcal{T}_{h}^{i}}\left\|\eta_{i}\right\|_{H^{j}(K)}^{2} \lesssim h^{4-2 j}\left|E_{i} v_{i}\right|_{H^{2}\left(\Omega_{h}^{i}\right)}^{2} \lesssim h^{4-2 j}|v|_{H^{2}\left(\Omega_{i}\right)}^{2} .
$$

Now we use (4.15) and (4.16) to bound each term of $\left\|v-I_{h} v\right\|_{V}$ separately. First, it is easy to see

$$
\sum_{K \in \mathcal{T}_{h}^{i}}\left\|\sqrt{a_{i}} \nabla \eta_{i}\right\|_{L^{2}\left(K \cap \Omega_{i}\right)}^{2} \lesssim a_{i} h^{2}\left|E_{i} v_{i}\right|_{H^{2}\left(\Omega_{h}^{i}\right)}^{2} \lesssim a_{i} h^{2}|v|_{H^{2}\left(\Omega_{i}\right)}^{2}
$$

Further, using the fact $\{a\}_{w} \leq 2 a_{i}$ and Lemma 3.3, we obtain

$$
\begin{aligned}
& \frac{\{a\}_{w}}{h} \sum_{K \in G_{h}^{\Gamma}}\|[\eta]\|_{L^{2}\left(\Gamma_{K}\right)}^{2} \lesssim \frac{\{a\}_{w}}{h} \sum_{i=1}^{2} \sum_{K \in G_{h}^{\Gamma}}\left\|\eta_{i}\right\|_{L^{2}\left(\Gamma_{K}\right)}^{2} \\
\lesssim & \sum_{i=1}^{2} \frac{a_{i}}{h} \sum_{K \in G_{h}^{\Gamma}}\left(h_{K}^{-1}\left\|\eta_{i}\right\|_{L^{2}(K)}^{2}+\left\|\eta_{i}\right\|_{L^{2}(K)}\left\|\nabla \eta_{i}\right\|_{L^{2}(K)}\right) \\
\lesssim & \sum_{i=1}^{2} a_{i} h^{2}|v|_{H^{2}\left(\Omega_{i}\right)}^{2} .
\end{aligned}
$$

Likewise, applying the fact $\frac{a_{i}^{2} w_{i}^{2}}{\{a\}_{w}} \leq \frac{1}{2} a_{i}$ and Lemma 3.3, we obtain

$$
\begin{aligned}
& \frac{h}{\{a\}_{w}} \sum_{K \in G_{h}^{\Gamma}}\left\|\{a \nabla \eta \cdot \mathbf{n}\}_{w}\right\|_{L^{2}\left(\Gamma_{K}\right)}^{2} \leq \sum_{i=1}^{2} \sum_{K \in G_{h}^{\Gamma}} a_{i} h\left\|\nabla \eta_{i} \cdot \mathbf{n}\right\|_{L^{2}\left(\Gamma_{K}\right)}^{2} \\
\lesssim & \sum_{i=1}^{2} \sum_{K \in G_{h}^{\Gamma}} a_{i} h\left(h_{K}^{-1}\left\|\nabla \eta_{i}\right\|_{L^{2}(K)}^{2}+\left\|\nabla \eta_{i}\right\|_{L^{2}(K)}\left|\nabla \eta_{i}\right|_{H^{1}(K)}\right) \\
\lesssim & \sum_{i=1}^{2} a_{i} h^{2}|v|_{H^{2}\left(\Omega_{i}\right)}^{2} .
\end{aligned}
$$

Similarly, the following inequality is obtained by the triangle and standard trace inequalities

$$
\sum_{i=1}^{2} \sum_{e \in \varepsilon_{h}^{\Gamma, i}} a_{i}|e|\|[\nabla \eta]\|_{L^{2}(e)}^{2} \lesssim \sum_{i=1}^{2} a_{i} h^{2}|v|_{H^{2}\left(\Omega_{i}\right)}^{2}
$$

For any $\widetilde{e} \in \varepsilon_{h}^{i, c u t}$, suppose that $e=\partial K_{l} \cap \partial K_{r}, K_{l}, K_{r} \in \mathcal{T}_{h}^{i}$ and $\widetilde{e} \subseteq e$. Then using 
Lemma 3.4, we have

$$
\begin{aligned}
|\widetilde{e}|^{-1}\left\|\left[\eta_{i}\right]\right\|_{L^{2}(\widetilde{e})}^{2} & \lesssim|\widetilde{e}|^{-1} \sum_{j=l, r}\left\|\left.\eta_{i}\right|_{K_{j}}\right\|_{L^{2}(\widetilde{e})}^{2} \\
& \lesssim \sum_{j=l, r}\left(\frac{1}{h_{K_{j}}^{2}}\left\|\eta_{i}\right\|_{L^{2}\left(K_{j}\right)}^{2}+\left\|\nabla \eta_{i}\right\|_{L^{2}\left(K_{j}\right)}^{2}+h_{K_{j}}^{2}\left|\nabla \eta_{i}\right|_{H^{1}\left(K_{j}\right)}^{2}\right) \\
& \lesssim \sum_{j=l, r} h_{K_{j}}^{2}\left|E_{i}\left(\left.v\right|_{\Omega_{i}}\right)\right|_{H^{2}\left(K_{j}\right)}^{2} .
\end{aligned}
$$

Hence

$$
\sum_{\widetilde{e} \in \varepsilon_{h}^{i, c u t}} a_{i}|\widetilde{e}|^{-1}\left\|\left[\eta_{i}\right]\right\|_{L^{2}(\widetilde{e})}^{2} \lesssim a_{i} h^{2}\left|E_{i}\left(\left.v\right|_{\Omega_{i}}\right)\right|_{H^{2}\left(\Omega_{h}^{i}\right)}^{2} \lesssim a_{i} h^{2}|v|_{H^{2}\left(\Omega_{i}\right)}^{2} .
$$

Using the triangle inequality and standard trace inequality, we have

$$
\begin{aligned}
& \sum_{\widetilde{e} \in \varepsilon_{h}^{i, c u t}} a_{i}|\widetilde{e}|\left\|\left[\nabla \eta_{i} \cdot \mathbf{n}_{\widetilde{e}}\right]\right\|_{L^{2}(\widetilde{e})}^{2} \\
\leq & \sum_{\widetilde{e} \in \varepsilon_{h}^{i, c u t}} a_{i}|e|\left\|\left[\nabla \eta_{i} \cdot \mathbf{n}_{\widetilde{e}}\right]\right\|_{L^{2}(e)}^{2} \\
\lesssim & \sum_{\widetilde{e} \in \varepsilon_{h}^{i, c u t}} a_{i}|e| \sum_{j=l, r}\left(|e|^{-1}\left\|\nabla \eta_{i}\right\|_{L^{2}\left(K_{j}\right)}^{2}+|e|\left|\nabla \eta_{i}\right|_{H^{1}\left(K_{j}\right)}^{2}\right) \\
\lesssim & a_{i} h^{2}|v|_{H^{2}\left(\Omega_{i}\right)}^{2} .
\end{aligned}
$$

Similarly,

$$
\sum_{\widetilde{e} \in \varepsilon_{h}^{i, c u t}} a_{i}|\widetilde{e}|\left\|\left\{\nabla \eta_{i} \cdot \mathbf{n}_{\widetilde{e}}\right\}_{k}\right\|_{L^{2}(\widetilde{e})}^{2} \lesssim a_{i} h^{2}|v|_{H^{2}\left(\Omega_{i}\right)}^{2} .
$$

Finally, from (4.17)-(4.23), it follows the result immediately.

We will give a priori error estimates in the energy and $L^{2}$ norms for our method.

Theorem 4.1. Let $u$ be the solution to problem (1.1) satisfying $u \in H^{2}\left(\Omega_{1} \cup \Omega_{2}\right)$ and $u_{h} \in V_{h}$ be its nonconforming extended finite element approximation solution of (2.2). Suppose that $\gamma_{i}, i=0,1,2$ are large enough and Assumptions (A1)-(A3) hold. Then the following inequality holds

$$
\left\|u-u_{h} \mid\right\| \lesssim h\left(\sqrt{a_{1}}\|\nabla u\|_{H^{1}\left(\Omega_{1}\right)}+\sqrt{a_{2}}|| \nabla u \|_{H^{1}\left(\Omega_{2}\right)}\right) .
$$

Proof. Note that $\left\|u-u_{h}|\|\leq\|| u-I_{h} u\right\|\left|+\left\|I_{h} u-u_{h}\right\|\right|$. Since $I_{h} u-u_{h} \in V_{h}$, from the coercivity and continuity of $A_{h}(\cdot, \cdot)$, we have

$$
\begin{aligned}
\left\|I_{h} u-u_{h}\right\|^{2} & \lesssim A_{h}\left(I_{h} u-u_{h}, I_{h} u-u_{h}\right) \\
& =A_{h}\left(I_{h} u-u, I_{h} u-u_{h}\right)+A_{h}\left(u-u_{h}, I_{h} u-u_{h}\right) \\
& \lesssim\left\|I_{h} u-u\right\|\left\|_{V}\right\| I_{h} u-u_{h} \|+A_{h}\left(u-u_{h}, I_{h} u-u_{h}\right) .
\end{aligned}
$$


Hence, we obtain

$$
\left\|\left|u-u_{h}\right|\right\| \lesssim\left\|u-I_{h} u\right\|_{V}+\sup _{v_{h} \neq 0 \in V_{h}} \frac{A_{h}\left(u-u_{h}, v_{h}\right)}{\left\|\left|v_{h}\right|\right\|} .
$$

From Lemma 4.3, it follows that

$$
\left\|u-\left.I_{h} u\left|\|_{V}^{2} \lesssim \sum_{i=1}^{2} a_{i} h^{2}\right| u\right|_{H^{2}\left(\Omega_{i}\right)} ^{2}\right.
$$

Thus, we only need to estimate the inconsistency error. From (2.5), it follows that

$$
A_{h}\left(u-u_{h}, v_{h}\right)=A_{h}\left(u, v_{h}\right)-L\left(v_{h}\right)=\sum_{i=1}^{2} \sum_{e \in \varepsilon_{h}^{i, n c}} \int_{e} a_{i} \nabla u \cdot \mathbf{n}_{e}\left[v_{h}\right]
$$

Let $\bar{v}=\frac{1}{|e|} \int_{e} v$. Then we have

$$
\int_{e} a_{i} \nabla u \cdot \mathbf{n}_{e}\left[v_{h}\right]=\int_{e} a_{i}\left(\nabla u \cdot \mathbf{n}_{e}-\overline{\nabla u \cdot \mathbf{n}_{e}}\right)\left[v_{h}-\overline{v_{h}}\right]
$$

where we have used that $\int_{e}\left[v_{h}\right]=0$ for $v_{h} \in V_{h}$.

Note that $\bar{v}$ is the $L^{2}$ projection of $v$ to the space of constant polynomials. Using the error estimate for polynomial projection and the standard error estimate on interpolation of Sobolev spaces (see [40]), we have the following inequality

$$
\|v-\bar{v}\|_{L^{2}(e)} \leq C h^{\frac{1}{2}}\|v\|_{H^{\frac{1}{2}(e)}} .
$$

Further, applying the Poincaré inequality, we have

$$
\|v-\bar{v}\|_{L^{2}(e)} \leq C|e|\|\nabla v\|_{L^{2}(e)} .
$$

Since $e \in \varepsilon_{h}^{i, n c}$ is the non-cut edge, there are three cases.

Case 1: $e=\partial K_{l} \cap \partial K_{r}, K_{l}, K_{r} \in \mathcal{T}_{h}$ are totally contained in $\Omega_{i}$. For (4.28), using the Cauchy-Schwarz inequality, (4.29) and (4.30), we have

$$
\begin{aligned}
\int_{e} a_{i} \nabla u \cdot \mathbf{n}_{e}\left[v_{h}\right] & \leq a_{i}|| \nabla u \cdot \mathbf{n}_{e}-\overline{\nabla u \cdot \mathbf{n}_{e}}\left\|_{L^{2}(e)}\right\|\left[v_{h}-\overline{v_{h}}\right] \|_{L^{2}(e)} \\
& \lesssim h^{\frac{1}{2}} a_{i}\left\|\nabla u \cdot \mathbf{n}_{e}\right\|_{H^{\frac{1}{2}(e)}}\left(\sum_{j=l, r}|e|\left\|\left.\nabla v_{h}\right|_{K_{j}}\right\|_{L^{2}(e)}\right) .
\end{aligned}
$$


Further, using the definition of $\|\cdot\|_{H^{\frac{1}{2}(e)}}$ and the fact that triangulations is conforming, quasi-uniform and regular, we have

$$
\begin{aligned}
\int_{e} a_{i} \nabla u \cdot \mathbf{n}_{e}\left[v_{h}\right] & \left.\lesssim h \sqrt{a_{i}}|| \nabla u\left\|_{H^{1}\left(K_{l} \cup K_{r}\right)} \sum_{j=l, r} \sqrt{a_{i}}|e|^{1 / 2}\right\| \nabla v_{h}\right|_{K_{j}} \|_{L^{2}(e)} \\
& \lesssim h \sqrt{a_{i}}\|\nabla u\|_{H^{1}\left(K_{l} \cup K_{r}\right)}\left(\sum_{j=l, r} \frac{|e|}{\sqrt{\left|K_{j}\right|}}\left\|\sqrt{a_{i}} \nabla v_{h}\right\|_{L^{2}\left(K_{j}\right)}\right) \\
& \lesssim h \sqrt{a_{i}}\|\nabla u\|_{H^{1}\left(K_{l} \cup K_{r}\right)}\left(\sum_{j=l, r}\left\|\sqrt{a_{i}} \nabla v_{h}\right\|_{L^{2}\left(K_{j}\right)}\right) \\
& \leq h \sqrt{a_{i}}\|\nabla u\|_{H^{1}\left(K_{l} \cup K_{r}\right)}\left\|v_{h} \mid\right\| .
\end{aligned}
$$

Case 2: $e=\partial K_{l} \cap \partial K_{r}, K_{l}, K_{r} \in \mathcal{T}_{h}$, where only one of the two elements is the cut element. Without loss of the generality, we assume that $K_{l}$ is contained in $\Omega_{i}$ and $K_{r} \in G_{h}^{\Gamma}$. Similarly,

$$
\begin{aligned}
& \int_{e} a_{i} \nabla u \cdot \mathbf{n}_{e}\left[v_{h}\right] \lesssim h \sqrt{a_{i}}|| \nabla u \|_{H^{1}\left(K_{l}\right)}\left(\sum_{j=l, r} \sqrt{a_{i}}|e|^{1 / 2}\left\|\left.\nabla v_{h}\right|_{K_{j}}\right\|_{L^{2}(e)}\right) \\
\lesssim & h \sqrt{a_{i}}\|\nabla u\|_{H^{1}\left(K_{l}\right)} \sqrt{a_{i}}|e|^{1 / 2}\left(\left\|\left.\nabla v_{h}\right|_{K_{l}}\right\|_{L^{2}(e)}+\left\|\left.\nabla v_{h}\right|_{K_{l}} \pm\left[\nabla v_{h}\right]\right\|_{L^{2}(e)}\right) \\
\lesssim & h \sqrt{a_{i}}\|\nabla u\|_{H^{1}\left(K_{l}\right)}\left(\left\|\sqrt{a_{i}} \nabla v_{h}\right\|_{L^{2}\left(K_{l}\right)}+|e|^{1 / 2} \sqrt{a_{i}}\left\|\left[\nabla v_{h}\right]\right\|_{L^{2}(e)}\right) \\
\lesssim & h \sqrt{a_{i}}\|\nabla u\|_{H^{1}\left(K_{l}\right)}\left\|\left|v_{h}\right|\right\| .
\end{aligned}
$$

Case 3: $e \in \varepsilon_{h}^{2, n c}$ and $e=\partial K \cap \partial \Omega, K \in \mathcal{T}_{h}^{2}$ (see Assumption (A3)). Similarly,

$$
\begin{aligned}
\int_{e} a_{i} \nabla u \cdot \mathbf{n}_{e}\left[v_{h}\right] & =\int_{e} a_{i}\left(\nabla u \cdot \mathbf{n}_{e}-\overline{\nabla u \cdot \mathbf{n}_{e}}\right)\left(v_{h}-\overline{v_{h}}\right) \\
& \lesssim h \sqrt{a_{2}}\|\nabla u\|_{H^{1}(K)}\left\|\mid v_{h}\right\|
\end{aligned}
$$

where we have used the fact that $\int_{e \cap \partial \Omega} v_{h}=0$ for $v \in V_{h}$.

Therefore,

$$
A_{h}\left(u-u_{h}, v_{h}\right) \lesssim\left(\sum_{i=1}^{2} h \sqrt{a_{i}}\|\nabla u\|_{H^{1}\left(\Omega_{i}\right)}\right)\left\|\mid v_{h}\right\| \| .
$$

Then, using (4.25), (4.26) and (4.35) yields the result. The proof is completed.

We now give the $L^{2}$ norm estimate with the technique of duality argument.

Theorem 4.2. Under the same conditions of Theorem 4.1, there holds

$$
\left\|u-u_{h}\right\|_{L^{2}(\Omega)} \lesssim h^{2} a_{\min }^{-1 / 2}\left(\sum_{i=1}^{2} \sqrt{a_{i}}\|\nabla u\|_{H^{1}\left(\Omega_{i}\right)}\right) .
$$


Remark 4.1. Suppose that $g_{D}=g_{N}=0$. Then there holds the following regularity estimate for the interface problem $[4,41]$ :

$$
\left|a_{i} u\right|_{H^{j}\left(\Omega_{i}\right)} \lesssim\|f\|_{L^{2}(\Omega)}, \quad i, j=1,2
$$

Thus, from (4.36), we conclude the following error bound for $L^{2}$-norm :

$$
\left\|u-u_{h}\right\|_{L^{2}(\Omega)} \lesssim h^{2} a_{\min }^{-1}\|f\|_{L^{2}(\Omega)},
$$

which does not depend on the contrast of the diffusion coefficient.

Proof. Let us consider an auxiliary problem: for $u-u_{h} \in L^{2}(\Omega)$, find $z \in H^{2}\left(\Omega_{1} \cup \Omega_{2}\right)$ such that

$$
\begin{cases}-\nabla \cdot(a(x) \nabla z)=u-u_{h} & \text { in } \Omega_{1} \cup \Omega_{2}, \\ {[z]=0, \quad[(a(x) \nabla z) \cdot \mathbf{n}]=0} & \text { on } \Gamma, \\ z=0 & \text { on } \partial \Omega .\end{cases}
$$

The solution satisfies the following regularity estimate (see $[4,41])$ :

$$
\left|a_{i} z\right|_{H^{j}\left(\Omega_{i}\right)} \lesssim\left\|u-u_{h}\right\|_{L^{2}(\Omega)}, \quad i, j=1,2 .
$$

Multiplying the equation of (4.37) by $u$, integrating on each subdomain $\Omega_{i}$, and using integration by parts, we obtain

$$
\begin{aligned}
\left(u-u_{h}, u\right) & =\sum_{i=1}^{2} \int_{\Omega_{i}} a_{i} \nabla z \cdot \nabla u-\int_{\Gamma}[a \nabla z \cdot \mathbf{n} u] \\
& =\sum_{i=1}^{2} \int_{\Omega_{i}} a_{i} \nabla z \cdot \nabla u-\int_{\Gamma}\{a \nabla z \cdot \mathbf{n}\}_{w}[u] .
\end{aligned}
$$

Here we have used the formula $[v w]=\{v\}_{w}[w]+[v]\{w\}^{w}$ and the fact that $[a \nabla z \cdot \mathbf{n}]=$ 0 . Therefore, using the definition of $A_{h}(\cdot, \cdot)$, and the facts that $[z]=0$ on $\Gamma$, and $[u]=0$, $[z]=0,\left[\nabla u \cdot \mathbf{n}_{\tilde{e}}\right]=\left[\nabla z \cdot \mathbf{n}_{\tilde{e}}\right]=0$ on $\widetilde{e} \in \varepsilon_{h}^{i, c u t}$, and $[\nabla u]=[\nabla z]=0$ on $e \in \varepsilon_{h}^{\Gamma, i}$, we have

$$
\left(u-u_{h}, u\right)=A_{h}(z, u) .
$$

Further, let $z_{h} \in V_{h}$ be the solution of the nonconforming extended finite element approximation of $z$ which satisfies

$$
A_{h}\left(z_{h}, v_{h}\right)=\left(u-u_{h}, v_{h}\right), \quad \forall v_{h} \in V_{h} .
$$

Hence

$$
\left\|u-u_{h}\right\|_{L^{2}(\Omega)}^{2}=\left(u-u_{h}, u\right)-\left(u-u_{h}, u_{h}\right)=A_{h}(z, u)-A_{h}\left(z_{h}, u_{h}\right) .
$$

Noticing that the bilinear form $A_{h}(\cdot, \cdot)$ is symmetric, from (4.40), we have

$$
\begin{aligned}
& \left\|u-u_{h}\right\|_{L^{2}(\Omega)}^{2}=A_{h}\left(z-z_{h}, u\right)+A_{h}\left(z_{h}, u-u_{h}\right) \\
= & A_{h}\left(z-z_{h}, u-I_{h} u\right)+A_{h}\left(z-z_{h}, I_{h} u\right)+A_{h}\left(u-u_{h}, z_{h}\right) .
\end{aligned}
$$


From continuity of $A_{h}(\cdot, \cdot)$, it follows that

$$
A_{h}\left(z-z_{h}, u-I_{h} u\right) \lesssim\left\|\mid z-z_{h}\right\|_{V}\left\|u-I_{h} u\right\|_{V} .
$$

Using the triangle inequality and (4.13), we have

$$
\begin{aligned}
\|\left.\left|z-z_{h}\right|\right|_{V} & \leq\left\|\left|z-I_{h} z\right|\right\|_{V}+\left\|\left|I_{h} z-z_{h}\right|\right\|_{V} \\
& \lesssim\left\||| z-I_{h} z\left|\left\|_{V}+\right\|\right| I_{h} z-z_{h} \mid\right\| .
\end{aligned}
$$

Further, from the proof of Theorem 4.1, we have

$$
\left\|I_{h} z-z_{h}\left|\left\|\lesssim h \sum_{i=1}^{2} \sqrt{a_{i}}|| \nabla z\right\|_{H^{1}\left(\Omega_{i}\right)} .\right.\right.
$$

Thus, from (4.42), (4.43), (4.38), and Lemma 4.3, it follows that

$$
\left\|\left|z-z_{h}\right|\right\|_{V} \lesssim h \sum_{i=1}^{2} \sqrt{a_{i}}\|\nabla z\|_{H^{1}\left(\Omega_{i}\right)} \lesssim a_{\min }^{-1 / 2} h\left\|u-u_{h}\right\|_{L^{2}(\Omega)} .
$$

Hence,

$$
A_{h}\left(z-z_{h}, u-I_{h} u\right) \lesssim a_{\min }^{-1 / 2} h^{2}\left(\sum_{i=1}^{2} \sqrt{a_{i}}|u|_{H^{2}\left(\Omega_{i}\right)}\right)\left\|u-u_{h}\right\|_{L^{2}(\Omega)} .
$$

For the second term of the right-hand side of (4.41), from (4.27) and (4.28), we have

$$
\begin{aligned}
A_{h}\left(z-z_{h}, I_{h} u\right) & =\sum_{i=1}^{2} \sum_{e \in \varepsilon_{h}^{i, n c}} \int_{e} a_{i} \nabla z \cdot \mathbf{n}_{e}\left[I_{h} u\right] \\
& =\sum_{i=1}^{2} \sum_{e \in \varepsilon_{h}^{i, n c}} \int_{e} a_{i}\left(\nabla z \cdot \mathbf{n}_{e}-\overline{\nabla z \cdot \mathbf{n}_{e}}\right)\left[I_{h} u-u\right] .
\end{aligned}
$$

Thus, using the Cauchy-Schwarz inequality, (4.29), trace inequality and (4.16), we have

$$
\begin{aligned}
A_{h}\left(z-z_{h}, I_{h} u\right) & \leq \sum_{i=1}^{2} \sum_{e \in \varepsilon_{h}^{i, n c}} a_{i}\left\|\nabla z \cdot \mathbf{n}_{e}-\overline{\nabla z \cdot \mathbf{n}_{e}}\right\|_{L^{2}(e)}\left\|\left[I_{h} u-u\right]\right\|_{L^{2}(e)} \\
& \lesssim \sum_{i=1}^{2} \sum_{e \in \varepsilon_{h}^{i, n c}} a_{i} h^{\frac{1}{2}}\left\|\nabla z \cdot \mathbf{n}_{e}\right\|_{H^{\frac{1}{2}(e)}}\left\|\left[I_{h} u-u\right]\right\|_{L^{2}(e)} \\
& \lesssim \sum_{i=1}^{2} a_{i} h^{2}\|\nabla z\|_{H^{1}\left(\Omega_{i}\right)}|u|_{H^{2}\left(\Omega_{i}\right)} \\
& \lesssim a_{\min }^{-1 / 2} h^{2}\left(\sum_{i=1}^{2} \sqrt{a_{i}}|u|_{H^{2}\left(\Omega_{i}\right)}\right)\left\|u-u_{h}\right\|_{L^{2}(\Omega)}
\end{aligned}
$$


Similarly, we have

$$
\begin{aligned}
A_{h}\left(u-u_{h}, I_{h} z\right) & \lesssim \sum_{i=1}^{2} a_{i} h^{2}\|\nabla u\|_{H^{1}\left(\Omega_{i}\right)}|z|_{H^{2}\left(\Omega_{i}\right)} \\
& \lesssim a_{\text {min }}^{-1 / 2} h^{2}\left(\sum_{i=1}^{2} \sqrt{a_{i}}\|\nabla u\|_{H^{1}\left(\Omega_{i}\right)}\right)\left\|u-u_{h}\right\|_{L^{2}(\Omega)} .
\end{aligned}
$$

Further, by the same argument as that of $A_{h}\left(z-z_{h}, u-I_{h} u\right)$, we obtain

$$
\begin{aligned}
A_{h}\left(u-u_{h}, z_{h}-I_{h} z\right) & \lesssim\left\|u-u_{h}\left|\left\|_ { V } \left|\left\|z_{h}-I_{h} z \mid\right\|\right.\right.\right.\right. \\
& \lesssim\left(\left\|u-I_{h} u\right\|_{V}+\left\|I_{h} u-u_{h} \mid\right\|\right)\left\|z_{h}-I_{h} z\right\| \\
& \lesssim a_{\min }^{-1 / 2} h^{2}\left(\sum_{i=1}^{2} \sqrt{a_{i}}\|\nabla u\|_{H^{1}\left(\Omega_{i}\right)}\right)\left\|u-u_{h}\right\|_{L^{2}(\Omega)} .
\end{aligned}
$$

Thus, from (4.47) and (4.48), we have

$$
\begin{aligned}
A_{h}\left(u-u_{h}, z_{h}\right) & =A_{h}\left(u-u_{h}, z_{h}-I_{h} z\right)+A_{h}\left(u-u_{h}, I_{h} z\right) \\
& \lesssim a_{\min }^{-1 / 2} h^{2}\left(\sum_{i=1}^{2} \sqrt{a_{i}}\|\nabla u\|_{H^{1}\left(\Omega_{i}\right)}\right)\left\|u-u_{h}\right\|_{L^{2}(\Omega)} .
\end{aligned}
$$

Finally, from (4.44), (4.46) and (4.49), it follows the result immediately.

\section{Condition number of the system matrix}

In this section, we will show that the condition number of the system matrix is independent of the location of the interface with respect to the mesh, provided that the mesh family is quasi-uniform.

From the definition of $V_{h}$, we have that for any $u_{h} \in V_{h}$, there exist $u_{h, i} \in V_{h}^{i}$ such that $\left.u_{h}\right|_{\Omega_{i}}=\left.u_{h, i}\right|_{\Omega_{i}}, i=1,2$, where $u_{h, i}=\sum_{l=1}^{N_{i}} u_{i}^{(l)} \phi_{l}^{i} \in V_{h}^{i}$, and $\phi_{l}^{i}$ is the standard nonconforming linear basis of $V_{h}^{i}$ and $N_{i}=\operatorname{dim} V_{h}^{i}$. We define vector $U_{i}=$ $\left(u_{i}^{(1)}, u_{i}^{(2)}, \cdots, u_{i}^{\left(N_{i}\right)}\right) \in \mathbb{R}^{N_{i}}, i=1,2$. Denote the standard Euclidean norm by $\left|U_{i}\right|_{N_{i}}$. Let $\mathcal{M}_{i}$ denotes the mass matrix defined by $\int_{\Omega_{h}^{i}} u_{h, i} v_{h, i}, i=1,2$ and $\mathcal{A}$ denotes the stiffness matrix associated with the bilinear form $A_{h}\left(u_{h}, v_{h}\right)$. The system matrix $\mathcal{A}$ has $\left(N_{1}+N_{2}\right) \times\left(N_{1}+N_{2}\right)$ dimensions, which is symmetric and positive definite. Since the mesh family $\left\{\mathcal{T}_{h}^{i}\right\}, i=1,2$ is quasi-uniform on each domain $\Omega_{h}^{i}$, the following inequalities hold (see [31])

$$
\mu_{i, \min }^{1 / 2}\left|U_{i}\right|_{N_{i}} \leq\left\|u_{h, i}\right\|_{L^{2}\left(\Omega_{h}^{i}\right)} \leq \mu_{i, \max }^{1 / 2}\left|U_{i}\right|_{N_{i}}, \quad i=1,2,
$$

where $\mu_{i, \min }$ and $\mu_{i, \max }$ denote the smallest and largest eigenvalues of $\mathcal{M}_{i}, i=1,2$. The condition number of the system matrix is defined by

$$
k(\mathcal{A}):=\|\mathcal{A}\|\left\|\mathcal{A}^{-1}\right\|,
$$


where

$$
\begin{aligned}
& \|\mathcal{A}\|:=\sup _{\left\{U=\left(U_{1}, U_{2}\right), U_{i} \in \mathbb{R}^{\left.\mathbb{N}_{i}, i=1,2\right\}}\right.} \frac{|\mathcal{A} U|_{N_{1}+N_{2}}}{|U|_{N_{1}+N_{2}}}, \\
& |U|_{N_{1}+N_{2}}^{2}:=\left|U_{1}\right|_{N_{1}}^{2}+\left|U_{2}\right|_{N_{2}}^{2} .
\end{aligned}
$$

We first prove the following Poincaré-Friedrichs inequality.

Lemma 5.1. For $v_{h} \in V_{h}$, if Assumptions (A1)-(A3) hold, then we have

$$
\left\|v_{h}\right\|_{L^{2}\left(\Omega_{h}^{1} \cup \Omega_{h}^{2}\right)} \lesssim a_{\min }^{-1 / 2}\left\|\left|v_{h}\right|\right\|
$$

where $\left\|v_{h}\right\|_{L^{2}\left(\Omega_{h}^{1} \cup \Omega_{h}^{2}\right)}^{2}:=\left\|v_{h, 1}\right\|_{L^{2}\left(\Omega_{h}^{1}\right)}^{2}+\left\|v_{h, 2}\right\|_{L^{2}\left(\Omega_{h}^{2}\right)}^{2}$.

Proof. We assume $\phi$ is solution of the following dual problem

$$
\begin{cases}-\nabla \cdot(a(x) \nabla \phi)=v_{h} & \text { in } \Omega_{1} \cup \Omega_{2} \\ {[\phi]=0, \quad[(a(x) \nabla \phi) \cdot \mathbf{n}]=0} & \text { on } \Gamma \\ \phi=0 & \text { on } \partial \Omega\end{cases}
$$

which satisfies the elliptic regularity estimate (see $[4,41]$ )

$$
a_{i}|\phi|_{H^{j}\left(\Omega_{i}\right)} \lesssim \|\left. v_{h}\right|_{L^{2}(\Omega)}, \quad i, j=1,2 .
$$

Multiplying the dual problem (5.2) with $v_{h}$ and integrating by parts, we have

$$
\begin{aligned}
\left\|v_{h}\right\|_{L^{2}(\Omega)}^{2}= & \sum_{i=1}^{2} \sum_{K \in \mathcal{T}_{h}^{i}}\left(-\nabla \cdot(a(x) \nabla \phi), v_{h}\right)_{K \cap \Omega_{i}} \\
= & \sum_{i=1}^{2} \sum_{K \in \mathcal{T}_{h}^{i}} \int_{K \cap \Omega_{i}} a_{i} \nabla \phi \cdot \nabla v_{h}-\sum_{K \in G_{h}^{\Gamma}} \int_{\Gamma_{K}}\{a \nabla \phi \cdot \mathbf{n}\}_{w}\left[v_{h}\right] \\
& -\sum_{i=1}^{2}\left(\sum_{\widetilde{e} \in \varepsilon_{h}^{i, c u t}} \int_{\widetilde{e}} a_{i} \nabla \phi \cdot \mathbf{n}_{\widetilde{e}}\left[v_{h}\right]+\sum_{e \in \varepsilon_{h}^{i, n c}} \int_{e} a_{i} \nabla \phi \cdot \mathbf{n}_{e}\left[v_{h}\right]\right) .
\end{aligned}
$$

Using the Cauchy-Schwarz inequality, we have

$$
\sum_{K \in \mathcal{T}_{h}^{i}} \int_{K \cap \Omega_{i}} a_{i} \nabla \phi \cdot \nabla v_{h} \leq \sqrt{a_{i}}|\phi|_{H^{1}\left(\Omega_{i}\right)}\left(\sum_{K \in \mathcal{T}_{h}^{i}}\left\|\sqrt{a_{i}} \nabla v_{h}\right\|_{L^{2}\left(K \cap \Omega_{i}\right)}^{2}\right)^{\frac{1}{2}},
$$


and

$$
\begin{aligned}
& \sum_{K \in G_{h}^{\Gamma}} \int_{\Gamma_{K}}\{a \nabla \phi \cdot \mathbf{n}\}_{w}\left[v_{h}\right] \\
\leq & \sqrt{\frac{h}{\{a\}_{w}}}\left\|\{a \nabla \phi \cdot \mathbf{n}\}_{w}\right\|_{L^{2}(\Gamma)}\left(\frac{\{a\}_{w}}{h} \sum_{K \in G_{h}^{\Gamma}}\left\|\left[v_{h}\right]\right\|_{L^{2}\left(\Gamma_{K}\right)}^{2}\right)^{\frac{1}{2}} \\
\lesssim & \sum_{i=1}^{2} \sqrt{a_{i} h}\left\|\left.\nabla \phi\right|_{\Omega_{i}} \cdot \mathbf{n}\right\|_{L^{2}(\Gamma)}\left(\frac{\{a\}_{w}}{h} \sum_{K \in G_{h}^{\Gamma}}\left\|\left[v_{h}\right]\right\|_{L^{2}\left(\Gamma_{K}\right)}^{2}\right)^{\frac{1}{2}} \\
\lesssim & \sum_{i=1}^{2} \sqrt{a_{i} h}\|\nabla \phi\|_{H^{1}\left(\Omega_{i}\right)}\left(\frac{\{a\}_{w}}{h} \sum_{K \in G_{h}^{\Gamma}}\left\|\left[v_{h}\right]\right\|_{L^{2}\left(\Gamma_{K}\right)}^{2}\right)^{\frac{1}{2}} .
\end{aligned}
$$

Here we have used $\frac{a_{i}^{2} w_{i}^{2}}{\{a\}_{w}} \leq \frac{a_{i}}{2}$ and $\left\|\left.\nabla \phi\right|_{\Omega_{i}}\right\|_{L^{2}(\Gamma)} \leq C_{\Gamma}\|\nabla \phi\|_{H^{1}\left(\Omega_{i}\right)}$. For $\widetilde{e} \in \varepsilon_{h}^{i, c u t}$, there exist two elements $K_{l}, K_{r} \in G_{h}^{\Gamma}$ such that $\widetilde{e}=\partial K_{l}^{i} \cap \partial K_{r}^{i}$ with $K_{j}^{i}=K_{j} \cap \Omega_{i}, j=l, r$. Use the trace inequality to obtain

$$
\begin{aligned}
& \int_{\widetilde{e}} a_{i} \nabla \phi \cdot \mathbf{n}_{\widetilde{e}}\left[v_{h}\right] \\
\lesssim & a_{i}\left(\sum_{j=l, r}|\widetilde{e}|^{1 / 2}\left\|\left.\nabla \phi\right|_{K_{j}} \cdot \mathbf{n}_{\widetilde{e}}\right\|_{L^{2}(\widetilde{e})}\right)|\widetilde{e}|^{-1 / 2}\left\|\left[v_{h}\right]\right\|_{L^{2}(\widetilde{e})} \\
\lesssim & a_{i}|\widetilde{e}|^{-1 / 2}\left\|\left[v_{h}\right]\right\|_{L^{2}(\widetilde{e})} \sum_{j=l, r}\left(\|\nabla \phi\|_{L^{2}\left(K_{j}^{i, *}\right)}+h_{K_{j}}|\nabla \phi|_{H^{1}\left(K_{j}^{i, *}\right)}\right),
\end{aligned}
$$

where $K_{j}^{i, *}=\Delta_{K} \cap \Omega_{i}$ and $\Delta_{K}=\cup_{T \in \mathcal{T}_{h}}\{K \cap T \neq \emptyset\}$. Then

$$
\sum_{\widetilde{e} \in \varepsilon_{h}^{i, c u t}} \int_{\widetilde{e}} a_{i} \nabla \phi \cdot \mathbf{n}_{\widetilde{e}}\left[v_{h}\right] \lesssim\left\|\sqrt{a_{i}} \nabla \phi\right\|_{H^{1}\left(\Omega_{i}\right)} \sum_{e \in \varepsilon_{h}^{i, c u t}} \sqrt{\frac{a_{i}}{|\widetilde{e}|}}\left\|\left[v_{h}\right]\right\|_{L^{2}(\widetilde{e})} .
$$

Moreover, similar to (4.32), (4.33) and (4.34), we have

$$
\sum_{e \in \varepsilon_{h}^{i, n c}} \int_{e} a_{i} \nabla \phi \cdot \mathbf{n}_{e}\left[v_{h}\right] \lesssim\left\|\sqrt{a_{i}} \nabla \phi\right\|_{H^{1}\left(\Omega_{i}\right)}\left\|v_{h}\right\| .
$$

In summary, from (5.4), (5.5), (5.6), (5.7) and the regularity estimate, the following estimate holds

$$
\left\|v_{h}\right\|_{L^{2}(\Omega)}^{2} \lesssim \sum_{i=1}^{2}\left\|\sqrt{a_{i}} \nabla \phi\right\|_{H^{1}\left(\Omega_{i}\right)}\left\|\mid v_{h}\right\|\left\|a_{\min }^{-1 / 2}\right\| v_{h}\left\|_{L^{2}(\Omega)}\right\| v_{h} \| .
$$


On the other hand, for any $K \in G_{h}^{\Gamma}$, we assume that $z \in \Omega_{i}$ be a vertex of $K$. According to Lemma 3.1, there exist an element $K_{z} \in \Delta_{z}$ and a constant $C_{z}$ such that $\left|K_{z} \cap \Omega_{i}\right| \geq$ $C_{z} h_{K_{z}}^{2}$. Then using the scaling argument and the fact that any two norms on a finite dimensional space are equivalent, we have

$$
\left\|v_{h, i}\right\|_{L^{2}(K)} \lesssim \frac{|K|}{\left|K_{z} \cap \Omega_{i}\right|}\left\|v_{h}\right\|_{L^{2}\left(K_{z} \cap \Omega_{i}\right)} \lesssim\left\|v_{h}\right\|_{L^{2}\left(K_{z} \cap \Omega_{i}\right)}
$$

which can prove that

$$
\left\|v_{h, i}\right\|_{L^{2}\left(\Omega_{h}^{i}\right)} \lesssim\left\|v_{h}\right\|_{L^{2}\left(\Omega_{i}\right)} .
$$

The lemma is proved by combining (5.8) with (5.9).

Lemma 5.2. For $v_{h} \in V_{h}$, if Assumptions (A1) and (A2) hold, then we have

$$
\left\|v_{h}\right\|_{V} \lesssim a_{\max }^{1 / 2} h^{-1}\left\|v_{h}\right\|_{L^{2}\left(\Omega_{h}^{1} \cup \Omega_{h}^{2}\right)}
$$

Proof. We bound each term of $\left\|\left|v_{h}\right|\right\|_{V}$ separately. First, using the inverse inequality, we can easily obtain

$$
\sum_{K \in \mathcal{T}_{h}^{i}}\left\|\sqrt{a_{i}} \nabla v_{h}\right\|_{L^{2}\left(K \cap \Omega_{i}\right)}^{2} \lesssim a_{i} h^{-2}\left\|v_{h, i}\right\|_{L^{2}\left(\Omega_{h}^{i}\right)}^{2} .
$$

Further, applying Lemma 3.3, $\{a\}_{w} \leq 2 a_{i}$ and the inverse inequality, we have

$$
\begin{aligned}
& \frac{\{a\}_{w}}{h} \sum_{K \in G_{h}^{\Gamma}}\left\|\left[v_{h}\right]\right\|_{L^{2}\left(\Gamma_{K}\right)}^{2} \lesssim \frac{\{a\}_{w}}{h} \sum_{i=1}^{2} \sum_{K \in G_{h}^{\Gamma}}\left\|v_{h, i}\right\|_{L^{2}\left(\Gamma_{K}\right)}^{2} \\
\lesssim & \sum_{i=1}^{2} \frac{a_{i}}{h} \sum_{K \in G_{h}^{\Gamma}}\left(h_{K}^{-1}\left\|v_{h, i}\right\|_{L^{2}(K)}^{2}+\left\|v_{h, i}\right\|_{L^{2}(K)}\left\|\nabla v_{h, i}\right\|_{L^{2}(K)}\right) \\
\lesssim & \sum_{i=1}^{2} a_{i} h^{-2}\left\|v_{h, i}\right\|_{L^{2}\left(\Omega_{h}^{i}\right)}^{2} .
\end{aligned}
$$

Similarly, from Lemma 3.3, the facts that $\frac{a_{i}^{2} \omega_{i}^{2}}{\{a\}_{w}} \leq \frac{1}{2} a_{i}$ and $\nabla v_{h, i}$ is a constant vector, and the inverse inequality, we obtain

$$
\begin{aligned}
& \frac{h}{\{a\}_{w}} \sum_{K \in G_{h}^{\Gamma}}\left\|\left\{a \nabla v_{h} \cdot \mathbf{n}\right\}_{w}\right\|_{L^{2}\left(\Gamma_{K}\right)}^{2} \leq \sum_{i=1}^{2} \sum_{K \in G_{h}^{\Gamma}} a_{i} h\left\|\nabla v_{h, i} \cdot \mathbf{n}\right\|_{L^{2}\left(\Gamma_{K}\right)}^{2} \\
\lesssim & h \sum_{i=1}^{2} \sum_{K \in G_{h}^{\Gamma}} a_{i} h_{K}^{-1}\left\|\nabla v_{h, i}\right\|_{L^{2}(K)}^{2} \lesssim \sum_{i=1}^{2} a_{i} h^{-2}\left\|v_{h, i}\right\|_{L^{2}\left(\Omega_{h}^{i}\right)}^{2} .
\end{aligned}
$$


Likewise, the following inequalities are obtained by the triangle, standard trace and inverse inequalities, and the fact $\nabla v_{h}$ is a piecewise constant vector

$$
\begin{aligned}
& \sum_{i=1}^{2} \sum_{e \in \varepsilon_{h}^{\Gamma, i}} a_{i}|e|\left\|\left[\nabla v_{h}\right]\right\|_{L^{2}(e)}^{2} \lesssim \sum_{i=1}^{2} a_{i} h^{-2}\left\|v_{h, i}\right\|_{L^{2}\left(\Omega_{h}^{i}\right)}^{2}, \\
& \sum_{\widetilde{e} \in \varepsilon_{h}^{i, c u t}} a_{i}|\widetilde{e}|\left\|\left[\nabla v_{h} \cdot \mathbf{n}_{\widetilde{e}}\right]\right\|_{L^{2}(\widetilde{e})}^{2} \lesssim \sum_{i=1}^{2} a_{i} h^{-2}\left\|v_{h, i}\right\|_{L^{2}\left(\Omega_{h}^{i}\right)}^{2}, \\
& \sum_{\widetilde{e} \in \varepsilon_{h}^{i, c u t}} a_{i}|\widetilde{e}|\left\|\left\{\nabla v_{h} \cdot \mathbf{n}_{\widetilde{e}}\right\}_{k}\right\|_{L^{2}(\widetilde{e})}^{2} \lesssim \sum_{i=1}^{2} a_{i} h^{-2}\left\|v_{h, i}\right\|_{L^{2}\left(\Omega_{h}^{i}\right)}^{2} .
\end{aligned}
$$

For any $\widetilde{e} \in \varepsilon_{h}^{i, c u t}$, suppose that $e=\partial K_{l} \cap \partial K_{r}, K_{l}, K_{r} \in \mathcal{T}_{h}^{i}$ and $\widetilde{e} \subseteq e$. From Lemma 3.4 and the fact that $\nabla v_{h, i}$ is a constant vector, we obtain

$$
\begin{aligned}
& |\widetilde{e}|^{-1}\left\|\left[v_{h}\right]\right\|_{L^{2}(\widetilde{e})}^{2} \lesssim|\widetilde{e}|^{-1} \sum_{j=l, r}\left\|v_{h, i}\right\|_{L^{2}(\widetilde{e})}^{2} \\
\lesssim & \sum_{j=l, r}\left(\frac{1}{h_{K_{j}}^{2}}\left\|v_{h, i}\right\|_{L^{2}\left(K_{j}\right)}^{2}+\left\|\nabla v_{h, i}\right\|_{L^{2}\left(K_{j}\right)}^{2}\right) .
\end{aligned}
$$

Then using the inverse inequality, we obtain

$$
\sum_{\widetilde{e} \in \varepsilon_{h}^{i, c u t}} a_{i}|\widetilde{e}|^{-1}\left\|\left[v_{h}\right]\right\|_{L^{2}(\widetilde{e})}^{2} \lesssim \sum_{i=1}^{2} a_{i} h^{-2}\left\|v_{h, i}\right\|_{L^{2}\left(\Omega_{h}^{i}\right)}^{2} .
$$

Hence

$$
\left\|\left|v_{h}\right|\right\|_{V} \lesssim h^{-1}\left(\sum_{i=1}^{2}\left\|\sqrt{a_{i}} v_{h, i}\right\|_{L^{2}\left(\Omega_{h}^{i}\right)}\right) \lesssim a_{\max }^{1 / 2} h^{-1}\left\|v_{h}\right\|_{L^{2}\left(\Omega_{h}^{1} \cup \Omega_{h}^{2}\right)} .
$$

This completes the proof.

With the above notations and inequalities, we now give the following theorem.

Theorem 5.1. Let $\left\{\mathcal{T}_{h}\right\}$ be a quasi-uniform family of triangulation of domain $\Omega$. Suppose that $\gamma_{i}, i=0,1,2$ are large enough and Assumptions (A1)-(A3) hold. Then the condition number of the system matrix $\mathcal{A}$ associated with the NXFEM (2.2) satisfies

$$
k(\mathcal{A}) \lesssim \frac{a_{\max }}{a_{\min }} \frac{1}{h^{2}}
$$

Proof. By the definition

$$
\begin{aligned}
|\mathcal{A} U|_{N_{1}+N_{2}} & =\sup _{\left\{W=\left(W_{1}, W_{2}\right), W_{i} \in \mathbb{R}^{\left.N_{i}, i=1,2\right\}}\right.} \frac{(\mathcal{A} U, W)}{|W|_{N_{1}+N_{2}}} \\
& =\sup _{\left\{W=\left(W_{1}, W_{2}\right), W_{i} \in \mathbb{R}^{N_{i}}, i=1,2\right\}} \frac{A_{h}\left(u_{h}, w_{h}\right)}{|W|_{N_{1}+N_{2}}} .
\end{aligned}
$$


Using the continuity of $A_{h}(\cdot, \cdot)$ and Lemma 5.2, we have

$$
\begin{aligned}
& A_{h}\left(u_{h}, w_{h}\right) \lesssim\left\|| | u _ { h } \left|\left\|_{V}|| w_{h} \mid\right\|_{V}\right.\right. \\
\lesssim & \left.a_{\max } h^{-2}|| u_{h}||_{L^{2}\left(\Omega_{h}^{1} \cup \Omega_{h}^{2}\right)}|| w_{h}\right|_{L^{2}\left(\Omega_{h}^{1} \cup \Omega_{h}^{2}\right)} \\
\lesssim & a_{\max } h^{-2} \widetilde{\mu_{\max }}|U|_{N_{1}+N_{2}}|W|_{N_{1}+N_{2}},
\end{aligned}
$$

where $\widetilde{\mu_{\max }}=\max \left\{\mu_{1, \max }, \mu_{2, \max }\right\}$ and we have applied the second inequality of (5.1). Then we have

$$
|\mathcal{A} U|_{N_{1}+N_{2}} \lesssim a_{\max } h^{-2} \widetilde{\mu_{\max }}|U|_{N_{1}+N_{2}}
$$

Hence,

$$
\begin{aligned}
\|\mathcal{A}\| & \sup _{\left\{U=\left(U_{1}, U_{2}\right), U_{i} \in \mathbb{R}^{N_{i}}, i=1,2\right\}} \frac{|\mathcal{A} U|_{N_{1}+N_{2}}}{|U|_{N_{1}+N_{2}}} \\
& \lesssim a_{\max } h^{-2} \widetilde{\mu_{\max }} .
\end{aligned}
$$

For the norm of the inverse, by the definition and coercivity of $A_{h}(\cdot, \cdot)$, it is easy to see that

$$
|\mathcal{A} U|_{N_{1}+N_{2}} \geq \frac{(\mathcal{A} U, U)}{|U|_{N_{1}+N_{2}}}=\frac{A_{h}\left(u_{h}, u_{h}\right)}{|U|_{N_{1}+N_{2}}} \gtrsim \frac{\|\left.\left|u_{h}\right|\right|^{2}}{|U|_{N_{1}+N_{2}}} .
$$

From Lemma 5.1, we have

$$
|\mathcal{A} U|_{N_{1}+N_{2}} \gtrsim \frac{\left.a_{\min }|| u_{h}\right|_{L^{2}\left(\Omega_{h}^{1} \cup \Omega_{h}^{2}\right)} ^{2}}{|U|_{N_{1}+N_{2}}} .
$$

Using the first inequality of (5.1), we obtain

$$
|\mathcal{A} U|_{N_{1}+N_{2}} \gtrsim a_{\min } \widetilde{\mu_{\min }}|U|_{N_{1}+N_{2}}
$$

where $\widetilde{\mu_{\min }}=\min \left\{\mu_{1, \min }, \mu_{2, \min }\right\}$. Since $U$ is arbitrary, it follows by setting $V=\mathcal{A} U$ that

$$
\begin{aligned}
\left\|\mathcal{A}^{-1}\right\| & =\sup _{\left\{V=\left(V_{1}, V_{2}\right), V_{i} \in \mathbb{R}^{N_{i}}, i=1,2\right\}} \frac{\left|\mathcal{A}^{-1} V\right|_{N_{1}+N_{2}}}{|V|_{N_{1}+N_{2}}} \\
& \lesssim a_{\min }^{-1} \widetilde{\mu_{\min }}-1
\end{aligned}
$$

Using the fact that $\left\{\mathcal{T}_{h}\right\}$ is quasi-uniform, we have $\frac{\widetilde{\mu_{\max }}}{\mu_{\min }} \lesssim 1$ from Theorem 9.8 of [39]. From (5.16), (5.18) and the definition of $k(\mathcal{A})$, it concludes the result. The proof is completed. 


\section{Numerical experiments}

In this section, we explore the properties of the methods presented in Section 4 and Section 5. The exact interface is approximated by a chain of straight lines and and the cut-cells are approximated by simple shapes (two triangles or a triangle and a quadrilateral). We summarize our experimental results in tables, displaying the following errors:

$$
\begin{aligned}
& e_{h}^{0}:=\left\|u-u_{h}\right\|_{L^{2}(\Omega)}, \quad e_{h}^{\infty}:=\left\|u-u_{h}\right\|_{L^{\infty}(\Omega)}, \\
& e_{h, \sqrt{a}}^{1}:=\left\|\sqrt{a}\left(\nabla u-\nabla u_{h}\right)\right\|_{L^{2}(\Omega)} .
\end{aligned}
$$

We consider the two dimensional domain $\Omega=(-1,1) \times(-1,1)$ with the curve interface $\Gamma=\left\{x \in \Omega: x_{1}^{2}+x_{2}^{2}=r^{2}\right\}$. Define $\Omega_{1}:=\left\{\mathbf{x} \in \Omega: x_{1}^{2}+x_{2}^{2}<r^{2}\right\}$ and $\Omega_{2}:=\Omega \backslash\left(\Omega_{1} \cup \Gamma\right)$. Consider the following exact solution:

$$
u= \begin{cases}\frac{x_{1}^{2}+x_{2}^{2}}{a_{1}} & \text { in } \Omega_{1}, \\ \frac{x_{1}^{2}+x_{2}^{2}}{a_{2}}+r^{2}\left(\frac{1}{a_{1}}-\frac{1}{a_{2}}\right) & \text { in } \Omega_{2},\end{cases}
$$

where $r=1 / 2$. A direct computation shows $f=-4$ and $g_{D}=g_{N}=0$. All the following results are obtained by our method with penalty parameters $\gamma_{0}=100$ and $\gamma_{1}=\gamma_{2}=100$.

In the first experiment, we test our theoretical results with the convergence of errors $e_{h}^{0}, e_{h, \sqrt{a}}^{1}$ and $e_{h}^{\infty}$. Set $a_{1}=1000$ and $a_{2}=1$. Five kinds of mesh size are chosen as $h=1 / 8,1 / 16,1 / 32,1 / 64,1 / 128$. The results are shown in Table 1 . It is observed that the convergence orders of the errors are optimal. Namely, the second order for $e_{h}^{0}$ and $e_{h}^{\infty}$, and the first order for $e_{h, \sqrt{a}}^{1}$, which demonstrate the theoretical results presented in Theorems 4.1 and 4.2 .

The second numerical test is designed to confirm the influence of the jump of the coefficient on the errors. To do this, we fix the mesh size $h=1 / 64$. The results for the errors $e_{h}^{0}, e_{h, \sqrt{a}}^{1}$ and $e_{h}^{\infty}$ are listed in Table 2 with $\left(a_{1}, a_{2}\right)=(10,1),\left(10^{2}, 1\right), \cdots,\left(10^{5}, 1\right)$. We can see that as $a_{\max } / a_{\min }$ goes larger, the change of the errors is very small. It

Table 1: Errors and convergence orders of the nonconforming approximation for problem (1.1) with $a_{1}=$ $1000, a_{2}=1$.

\begin{tabular}{||c|cc|cc|cc||}
\hline$h$ & $e_{h}^{0}$ & rate & $e_{h, \sqrt{a}}^{1}$ & rate & $e_{h}^{\infty}$ & rate \\
\hline $1 / 8$ & $4.9321 \mathrm{E}-02$ & & $2.7160 \mathrm{E}-01$ & & $8.1137 \mathrm{E}-02$ & \\
\hline $1 / 16$ & $1.1322 \mathrm{E}-02$ & 2.1231 & $1.0173 \mathrm{E}-01$ & 1.4168 & $1.8817 \mathrm{E}-02$ & 2.1083 \\
\hline $1 / 32$ & $2.3123 \mathrm{E}-03$ & 2.2917 & $4.2598 \mathrm{E}-02$ & 1.2558 & $4.1915 \mathrm{E}-03$ & 2.1665 \\
\hline $1 / 64$ & $4.2490 \mathrm{E}-04$ & 2.4442 & $1.9601 \mathrm{E}-02$ & 1.1198 & $9.1739 \mathrm{E}-04$ & 2.1919 \\
\hline $1 / 128$ & $9.2652 \mathrm{E}-05$ & 2.1972 & $9.6622 \mathrm{E}-03$ & 1.0205 & $2.1966 \mathrm{E}-04$ & 2.0623 \\
\hline
\end{tabular}




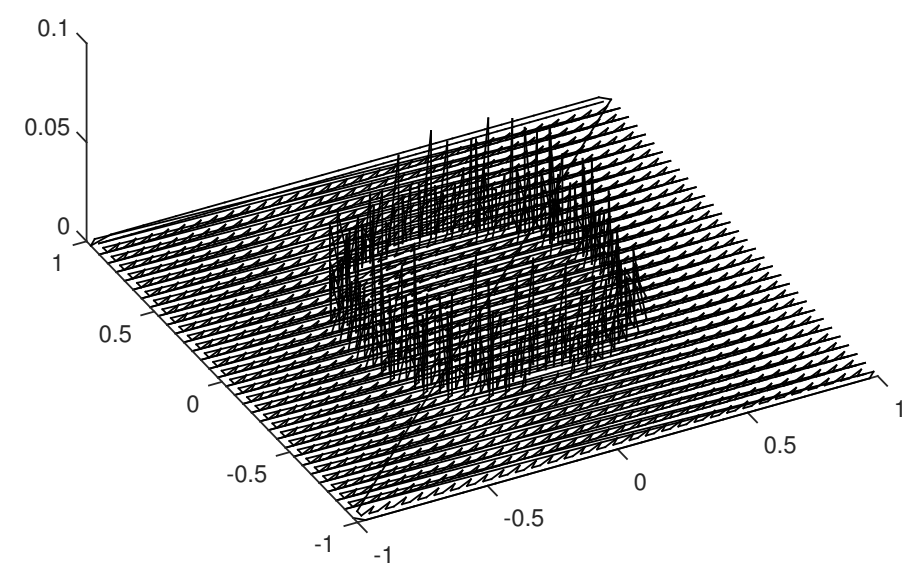

Figure 5: Elevation of the error for $h=1 / 32$.

indicates that the errors are convergent as $\frac{a_{\max }}{a_{\min }} \rightarrow \infty$, which means that they are all independent of the jump of the coefficient.

Further, local errors between the exact solution of (1.1) and the numerical solution of (2.2) on each cell with the mesh $h=1 / 32$ are shown in Fig. 5. We note that the errors are largest around the interface, that is to say, the errors are concentrated around the discontinuity. Based on this observation, it is suggested to use a fine mesh in the vicinity of the interface via the adaptive technique.

Finally, we give two tests to verify the theoretical result on the condition number of the system proved in Section 5. First, we give the relationship between the condition number and the mesh size. To do this, we fix $a_{1}=1000, a_{2}=1$, and change the values of $h$. Fig. 6 reports $\log$-log plot of $k(\mathcal{A})$ versus $1 / h$ with $h=\frac{1}{8}, \frac{1}{16}, \frac{1}{32}, \frac{1}{64}$. It can be seen that $k(\mathcal{A})=\mathcal{O}\left(h^{-2}\right)$ with the fixed value $\frac{a_{\max }}{a_{\min }}=1000$, which verifies the result of Theorem 5.1.

Further, we fix the mesh size $h=1 / 32$ to confirm the influence of the jump of the coefficients on the condition number. Fig. 7 shows $\log$-log plot of $k(\mathcal{A})$ versus $a_{1} / a_{2}$ with $\left(a_{1}, a_{2}\right)=(10,1),\left(10^{2}, 1\right), \cdots,\left(10^{6}, 1\right)$. It is shown that the condition number $k(\mathcal{A})$ depends on $\frac{a_{\max }}{a_{\min }}$ linearly and hence verifies Theorem 5.1 .

Table 2: Errors with $\left(a_{1}, a_{2}\right)=(10,1),\left(10^{2}, 1\right), \cdots,\left(10^{5}, 1\right)$ for $h=1 / 64$.

\begin{tabular}{||c|c|c|c|c||}
\hline$a_{1}$ & $a_{2}$ & $e_{h}^{0}$ & $e_{h, \sqrt{a}}^{1}$ & $e_{h}^{\infty}$ \\
\hline 10 & 1 & $4.1443 \mathrm{E}-04$ & $2.2971 \mathrm{E}-02$ & $8.6676 \mathrm{E}-04$ \\
\hline 100 & 1 & $4.2381 \mathrm{E}-04$ & $1.9932 \mathrm{E}-02$ & $9.0851 \mathrm{E}-04$ \\
\hline 1000 & 1 & $4.2490 \mathrm{E}-04$ & $1.9601 \mathrm{E}-02$ & $9.1739 \mathrm{E}-04$ \\
\hline 10000 & 1 & $4.2501 \mathrm{E}-04$ & $1.9568 \mathrm{E}-02$ & $9.1833 \mathrm{E}-04$ \\
\hline 100000 & 1 & $4.2502 \mathrm{E}-04$ & $1.9565 \mathrm{E}-02$ & $9.1843 \mathrm{E}-04$ \\
\hline
\end{tabular}




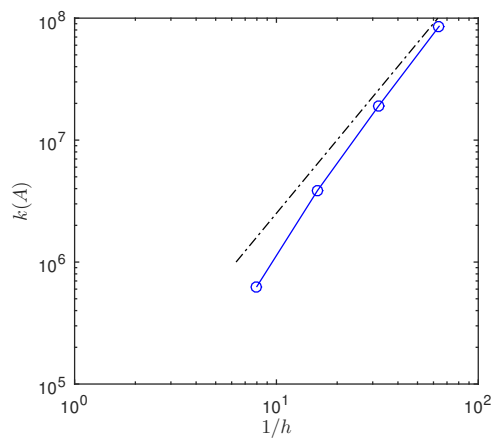

Figure 6: Log-log plot of $k(\mathcal{A})$ versus $1 / h\left(h=\frac{1}{8}, \frac{1}{16}, \frac{1}{32}, \frac{1}{64}\right)$ with $a_{1}=1000, a_{2}=1$. The dotted line gives the reference line of slope 2 .

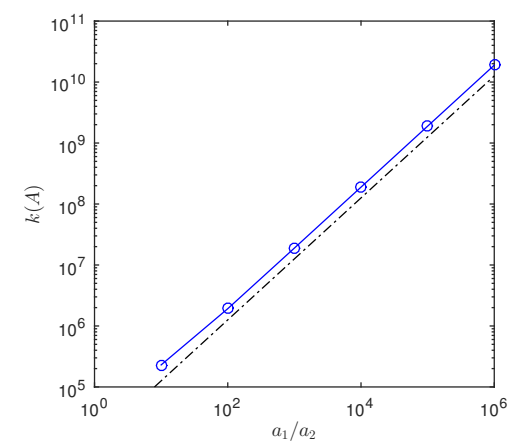

Figure 7: Log-log plot of $k(\mathcal{A})$ versus $a_{1} / a_{2}$ with $\left(a_{1}, a_{2}\right)=(10,1),\left(10^{2}, 1\right), \cdots,\left(10^{6}, 1\right)$ for $h=1 / 32$. The dotted line gives the reference line of slope 1 .

\section{Conclusions}

In this paper, we have proposed a nonconforming Nitsche's extended finite element method with the stabilization terms to solve the elliptic interface problems. The method allows the elements cut by the interface and defines the enriched nonconforming finite element space on each side of the interface by the standard nonconforming piecewise linear polynomials with the edge averages as degrees of freedom. We use the weighted average flux with so-called "harmonic weights", which are only related to the diffusion coefficients instead of that related to coefficients and subareas of the interface elements across the interface, to guarantee errors independent of the ratio of the discontinuous coefficients. Further, extra stabilization terms are added in the bilinear form to guarantee the stability and a well conditioning. A rigorously analysis shows that the errors of the proposed method are optimal and independent of the jump of the coefficients. Moreover, both the errors and the condition number of the system matrix do not rely on the interface position with respect to the mesh. Several numerical examples in two dimensions have been given to demonstrate the theoretical results. 
Acknowledgements The work of the second author was partially supported by the Natural Science Foundation of the Jiangsu Higher Institutions of China (No. 18KJB110015) and by No. GXL2018024. The work of the third author was partially supported by the the NSF of China grant No. 10971096, and by the Project Funded by the Priority Academic Program Development of Jiangsu Higher Education Institutions. The authors wish to thank two anonymous referees for many constructive suggestions and comments that lead to a great improvement of the paper.

\section{References}

[1] C. S. Peskin, Numerical analysis of blood flow in the heart, J. Comput. Phys., 25(3) (1977), pp. 220-252.

[2] R. LEVEQUE AND Z. LI, The immersed interface method for elliptic equations with discontinuous coefficients and singular sources, SIAM J. Numer. Anal., 31 (1994), pp. 1019-1044.

[3] R. P. Fedkiw, T. Aslam, B. Merriman and S. Osher, A non-oscillatory Eulerian approach to interfaces in multimaterial flows (the ghost fluid method), J. Comput. Phys., 152(2) (1999), pp. 457-492.

[4] C.-C. Chu, I. G. Graham And T.-Y. Hou, A new multiscale finite element method for high-contrast elliptic interface problems, Math. Comput., 79(272) (2010), pp. 1915-1955.

[5] Z. LI, The immersed interface method using a finite element formulation, Appl. Numer. Math., 27(3) (1998), pp. 253-267.

[6] Z. LI, T. LIN AND X. WU, New Cartesian grid methods for interface problems using the finite element formulation, Numer. Math., 96(1) (2003), pp. 61-98.

[7] Y. GONG, B. LI AND Z. LI, Immersed-interface finite-element methods for elliptic interface problems with nonhomogeneous jump conditions, SIAM J. Numer. Anal., 46(1) (2007/08), pp. 472-495.

[8] Y. GONG AND Z. LI, Immersed interface finite element methods for elasticity interface problems with non-homogeneous jump conditions, Numer. Math. Theory Methods Appl., 3(1) (2010), pp. 23-39.

[9] T. Lin, D. SHEEN AND X. ZHANG, A nonconforming immersed finite element method for elliptic interface problems, in: ArXiv:1510.00052, (2017).

[10] T. LIN, Q. YANG AND X. ZHANG, Partially penalized immersed finite element methods for parabolic interface problems, Numer. Methods Partial Differential Equations, 31(6) (2015), pp. 1925-1947.

[11] A. HansBo AND P. HANsBo, An unfitted finite element method, based on Nitsche's method, for elliptic interface problems, Comput. Methods Appl. Mech. Eng., 191(47-48) (2002), pp. 5537-5552.

[12] R. MAssuUng, An hp-error estimate for an unfitted discontinuous Galerkin method applied to elliptic interface problems, RWTH 300, IGPM Report, (2009).

[13] H. WU AND Y. XIAO, An unfitted hp-interface penalty finite element method for elliptic interface problems, in: arXiv: 1007.2893v1, (2010).

[14] E. WAdBro, S. ZAHEdi, G. KReiss AND M. BERGgren, A uniformly well-conditioned, unfitted nitsche method for interface problems, Bit Numer. Math., 53(3) (2013), pp. 791-820.

[15] F. WANG, Y. XIAO AND J. XU, High-order extended finite element methods for solving interface problems, in: arXiv: 1604.06171, (2016).

[16] E. Burman, J. GuZMÁn, M. A. SÁNCHEZ AND M. SARKIS, Robust flux error estimation of Nitsche's method for high contrast interface problems, IMA J. Numer. Anal., 38(2) (2018), 
pp. 646-668.

[17] E. BuRMAN AND P. Zunino, Numerical approximation of large contrast problems with the unfitted Nitsche method, in Frontiers in Numerical Analysis-Durham 2010, volume 85 of Lect. Notes Comput. Sci. Eng., pp. 227-282, Springer, Heidelberg, 2012.

[18] P. HuAnG, H. Wu AND Y. XIAO, An unfitted interface penalty finite element method for elliptic interface problems, Comput. Methods Appl. Mech. Eng., 323 (2017), pp. 439-460.

[19] J. HuANG AND J. Zou, A mortar element method for elliptic problems with discontinuous coefficients, IMA J. Numer. Anal., 22(4) (2002), pp. 549-576.

[20] Z. CHEN AND J. Zou, Finite element methods and their convergence for elliptic and parabolic interface problems, Numer. Math., 79(2) (1998), pp. 175-202.

[21] M. DRYJA, On discontinuous Galerkin methods for elliptic problems with discontinuous coefficients, Comput. Methods Appl. Math., 3(1) (2003), pp. 76-85.

[22] G. Guyomarc'H, C.-O. LeE And K. JeOn, A discontinuous Galerkin method for elliptic interface problems with application to electroporation, Commun. Numer. Methods Eng., 25(10) (2009), pp. 991-1008.

[23] E. BURMAN AND P. HANSBO, Interior-penalty-stabilized Lagrange multiplier methods for the finite-element solution of elliptic interface problems, IMA J. Numer. Anal., 30(3) (2010), pp. 870-885.

[24] J. Li, J. M. Melenk, B. Wohlmuth And J. Zou, Optimal a priori estimates for higher order finite elements for elliptic interface problems, Appl. Numer. Math., 60(1-2) (2010), pp. 19-37.

[25] N. Barrau, R. Becker, E. Dubach and R. Luce, A robust variant of NXFEM for the interface problem, C. R. Math. Acad. Sci. Paris, 350 (15-16) (2012), pp. 789-792.

[26] J. GuZMÁn, M. A. SÁNCHEZ AND M. SARKIS, A finite element method for high-contrast interface problems with error estimates independent of contrast, J. Sci. Comput., 73(1) (2017), pp. 330-365.

[27] P. HANSBo, M. G. LARSON AND S. ZAHEDi, A cut finite element method for a Stokes interface problem, Appl. Numer. Math., 85 (2014), pp. 90-114.

[28] L. Cattaneo, L. Formaggia, G. F. Iori, A. Scotti And P. Zunino, Stabilized extended finite elements for the approximation of saddle point problems with unfitted interfaces, Calcolo, 52(2) (2015), pp. 123-152.

[29] A. Massing, B. Schott And W. A. Wall, A stabilized Nitsche cut finite element method for the Oseen problem, Comput. Methods Appl. Mech. Eng., 328 (2018), pp. 262-300.

[30] E. Burman, Ghost penalty, C. R. Math. Acad. Sci. Paris, 348(21-22) (2010), pp. 12171220.

[31] E. BuRMAN AND P. HANSBO, Fictitious domain finite element methods using cut elements: II. A stabilized Nitsche method, Appl. Numer. Math., 62(4) (2012), pp. 328-341.

[32] P. Zunino, L. Cattaneo And C. M. Colciago, An unfitted interface penalty method for the numerical approximation of contrast problems, Appl. Numer. Math., 61(10) (2011), pp. 1059-1076.

[33] D. Y. KWAK, K. T. WEE AND K. S. CHANG, An analysis of broken $p_{1}$-nonconforming finite element method for interface problems, SIAM J. Numer. Anal., 48(6) (2009), pp. 21172134.

[34] D. Capatina, H. El-Otmany, D. Graebling and R. Luce, Extension of NXFEM to nonconforming finite elements, Math. Comput. Simulation, 137 (2017), pp. 226-245.

[35] D. Capatina, S. Delage Santacreu, H. El-Otmany and D. Graebling, Nonconforming finite element approximation of an elliptic interface problem with NXFEM, in: Thirteenth International Conference Zaragoza-Pau on Mathematics and Its Applications, Vol. 40 of 
Monogr. Mat. García Galdeano, Prensas Univ. Zaragoza, Zaragoza, 2016, pp. 43-52.

[36] Z. CAI, X. YE AND S. ZhANG, Discontinuous Galerkin finite element methods for interface problems: a priori and a posteriori error estimations, SIAM J. Numer. Anal., 49(5) (2011), pp. 1761-1787.

[37] M. DRYJA AND O. B. Widlund, Domain decomposition algorithms with small overlap, SIAM J. Sci. Comput., 15(3) (1994), pp. 604-620.

[38] D. Gilbarg And N. S. Trudinger, Elliptic Partial Differential Equations of Second Order, Springer-Verlag, 1983.

[39] A. ERn AND J. L. GUERmond, Theory and Practice of Finite Elements, Springer-Verlag, New York, 2004.

[40] V. Girault AND P.-A. RAVIART, Finite element methods for Navier-Stokes equations, Vol. 5 of Springer Series in Computational Mathematics, Springer-Verlag, Berlin, 1986, Theory and Algorithms.

[41] J. HUANG AND J. ZOU, Uniform a priori estimates for elliptic and static Maxwell interface problems, Discrete Contin. Dyn. Syst. Ser. B, 7(1) (2007), pp. 145-170.

[42] B. DEKA, A weak Galerkin finite element method for elliptic interface problems with polynomial reduction, Numer. Math. Theory Methods Appl., 11(3) (2018), pp. 655-672.

[43] H. ZHU, K. LIANG, G. HE AND J. YING, A splitting collocation method for elliptic interface problems, Numer. Math. Theory Methods Appl., 11(3) (2018), pp. 491-505.

[44] C. LIU AND C. HU, A second order ghost fluid method for an interface problem of the Poisson equation, Commun. Comput. Phys., 22(4) (2017), pp. 965-996.

[45] L. MU, J. WANG AND X. YE, A new weak Galerkin finite element method for elliptic interface problems, J. Comput. Phys., 325 (2016), pp. 157-173.

[46] J. A. Nitsche, Über ein Variationsprinzip zur Lösung Dirichlet-Problemn bei Verwendung von Teilräumen, die keinen Randbedingungen unteworfen sind, Abh. Math. Semin. Univ. Hambg., 36 (1971), pp. 9-15. 